\title{
Perturbations of Schwarzschild black holes in the Lorenz gauge: Formulation and numerical implementation
}

\author{
Leor Barack \\ School of Mathematics, University of Southampton, Southampton, SO17 1BJ, United Kingdom \\ Carlos O. Lousto \\ Department of Physics and Astronomy, and Center for Gravitational Wave Astronomy, The University of Texas at \\ Brownsville, Brownsville, Texas 78520, USA
}

(February 6, 2008)

\begin{abstract}
We reformulate the theory of Schwarzschild black hole perturbations in terms of the metric perturbation in the Lorenz gauge. In this formulation, each tensor-harmonic mode of the perturbation is constructed algebraically from 10 scalar functions, satisfying a set of 10 wavelike equations, which are decoupled at their principal parts. We solve these equations using numerical evolution in the time domain, for the case of a pointlike test particle set in a circular geodesic orbit around the black hole. Our code uses characteristic coordinates, and incorporates a constraint damping scheme. The axially-symmetric, odd-parity modes of the perturbation are obtained analytically. The approach developed here is especially advantageous in applications requiring knowledge of the local metric perturbation near a point particle; in particular, it offers a useful framework for calculations of the gravitational self force.
\end{abstract}

04.30.Db, 04.25.Nx, 04.25.-g, 04.70.Bw

\section{INTRODUCTION}

There has been much progress, over the past few years, on the problem of finding the "self force" (SF) correction to the geodesic motion of point particles in curved geometries [1,2]. This had strong motivation from the need to accurately model the orbital evolution in astrophysical binaries with extreme mass ratios - a key type of sources for the planned gravitational-wave detector LISA (the Laser Interferometer Space Antenna) [3]. Yet, despite the fact that a formal framework for calculations of the gravitational SF exists since quite a while ago [4-7], most actual computations so far have been restricted to toy models based on scalar or electromagnetic fields, with very little progress on the gravitational problem of relevance to LISA. What held progress up, mainly, is a certain gap within the standard glossary of techniques comprising black hole perturbation theory. Here we intend to supplement the necessary piece of perturbative technology needed to facilitate calculations of the gravitational SF. Although our motivation bears primarily on the SF problem, we expect the perturbation approach to be developed here to be useful in a wider class of problems in mathematical and numerical relativity.

What has been hindering calculations of the gravitational SF can be described, in most simple terms, as follows. The term "gravitational SF" refers to the effective local force exerted on a point mass particle through interaction with its own gravitational field. (The notion of a "point particle", in our general-relativistic context, builds on the clear separation of length scales in the extreme-mass-ratio inspiral problem-see, e.g., the discussion in [1].) Technically, the SF appears as a correction term, quadratic in the particle's mass, in the geodesic equation of motion. To obtain the SF one first needs to address the subtle issue of "regularization": telling the part of the particle's field whose back-reaction affects the motion, from a remaining, singular piece that can be safely "removed" without affecting the motion. A formal procedure for identifying the correct "regularizable" piece (for mass particles in any vacuum curved spacetime) has been established in works by Mino et al. [5], Detweiler and whiting [6], and others.

The above regularization procedure involves the introduction of a suitable local frame for the particle, and also the imposition of an appropriate gauge condition for the particle's field, the latter being treated as a perturbation over the fixed background of the massive black hole. Recall here that the metric perturbation (MP) is subject to a gauge freedom, associated with infinitesimal coordinate transformations. The full MP induced by the particle is gauge dependent, and so is the form of its singular, "regularizable" piece. This local piece is most conveniently constructed within the so-called "Lorenz gauge": an analogue of the familiar Lorenz gauge of electromagnetism, wherein the covariant divergence of the (trace-reversed) MP is taken to vanish [see Eq. (3) below]. The Lorenz gauge condition conforms with the isotropic form of the singularity very close to the particle (as viewed from a suitable local frame), and the Lorenz-gauge MP correctly reflects the "inverse-distance" behavior of this singularity. This local isotropy of the Lorenz gauge is an essential feature that should not be taken for granted: Expressed in a poorly chosen gauge, the 
physical point singularity may take a complicated form, that could render analysis intractable. Indeed, it has been shown that with the standard gauge choices commonly made in studies of black hole perturbations (see below) the physical point singularity can take a distorted form, and may even cease to be isolated [8].

Once the "correct" singular piece of the MP is identified, the construction of the SF proceeds by removing this piece from the full (retarded) field of the particle, and then calculating the force exerted on the particle by the remaining finite field. (The full field is obtained, in principle, by solving the perturbation equations with an energy-momentum source term representing the point particle.) A practical way to subtract the singular piece from the full field is offered by the mode sum scheme [7], in which one first decomposes the full MP into multipole harmonics (defined with respect to the background black hole geometry) and then carries out the subtraction mode by mode - thereby avoiding the need to deal with singular fields. The mode-by-mode subtraction scheme, like the original construction in $[4,5]$, is formulated in the Lorenz gauge, and relies on having at hand the full Lorenz-gauge MP near the particle.

Unfortunately, the standard toolkit of black hole perturbation theory does not include practical techniques or working tools for calculating the MP in the Lorenz gauge. Standard formulations of black hole perturbations employ other gauges, which are favored for their algebraic simplicity. A most commonly favored gauge choice for analyzing perturbations of spherically symmetric black hole spacetimes is the one introduced long ago by Regge and Wheeler (RW) [9] (and developed further by others, including Zerili [10,11] and Moncrief [12]). In the RW gauge, certain projections of the MP onto a tensor-harmonic basis, in a specific coordinate system, are taken to vanish. ${ }^{1}$ Another such "algebraic" gauge proven useful, is the one referred to as the radiation gauge [14], where one sets to zero the projection of the MP along a principle null direction of the background black hole geometry. Perturbations of the Kerr geometry have been studied almost exclusively through the powerful Teukolsky formalism [15], in which the perturbation is formulated in terms of the Weyl scalars, rather than the metric. A reconstruction procedure for the MP, out of the perturbation in the Weyl scalars, has been prescribed by Chrzanowski [14] (with later supplements by Wald [16] and Ori [17]). This reconstruction is formulated within the radiation gauge, and relies crucially on this choice of gauge.

Hence, we are in a situation where the singular, "removable" piece of the MP near the particle is given in the Lorenz gauge (which best reflects the symmetry of the point singularity), while the full field of the particle can only be calculated in the RW or radiation gauges (which bear on the symmetries of the black hole background). Much effort has been invested recently in trying to resolve this problematic issue. One approach has been to try reformulating the SF, and the subtraction scheme, entirely within the RW gauge (in the Schwarzschild background) $[18,19]$. Another approach incorporated an "intermediate" gauge, in which the MP admits the isotropic Lorenz-gauge form near the particle, but gradually approaches the form of the RW (or radiation) gauge as one moves away from the particle [8]. Neither approaches have gone very far, partly because of the difficulty in tackling the singular gauge transformations involved. The complicated singular nature of these gauge transformations reflects the fact that the RW/radiation gauges are far from being natural gauges to describe point particle singularities. For example, is has been demonstrated [8] that the radiation-gauge MP develops a singularity along an infinite ray emanating from the particle. Indeed, the only actual computation of the gravitational SF carried out so far [20,21] restricted to a special case where the RW-gauge MP happens to coincide with the Lorenz-gauge MP (the case of a strictly radial infall into a Schwarzschild black hole).

Of course, the above gauge problem would have never occurred, had we the right tools for calculating the full perturbation field of the particle in the Lorenz gauge. This would have allowed a direct approach to SF calculations, based entirely on the Lorenz gauge and avoiding the above gauge-related complications. In this work we begin developing such practical tools for analysis of Lorenz-gauge perturbations. This paper deals with MP of the Schwarzschild black hole. It formulates the Lorenz-gauge MP equations in a form accessible to numerical time-evolution treatment, and presents calculations of the Lorenz-gauge field in the example of a mass particle moving in a circular geodesic in the strong field of the black hole. The paper also discusses, in brief, how we might go about carrying out Lorenz-gauge calculations in the Kerr spacetime. This important task is currently subject to intensive study.

It may be worthwhile to summarize here, in bullet points, the main strengths of our Lorenz-gauge approach to black hole perturbations. The following points also give cues to the variety of problems where such an approach can prove useful.

- A regularization scheme for the SF in Kerr spacetime has only been prescribed in the Lorenz gauge. It is not clear yet how one goes about formulating and calculating the gravitational SF in other gauges. Having at hand

\footnotetext{
${ }^{1}$ There is an equivalent formulation of the RW gauge, which does not rely on a tensor-harmonic decomposition [8,13]. In this formulation one sets to zero certain combinations of the MP components and their derivatives, in a certain coordinate system.
} 
the Lorenz-gauge MP allows one, immediately, to compute the gravitational SF, without having to resort to cumbersome gauge adjustments.

- In our approach one solves directly for the MP components, without resorting to complicated reconstruction procedures. This feature becomes important in problems where knowledge of the Weyl scalars (or RW/Zerilli's variables, or Moncrief's variables) is insufficient, and direct access to the MP itself is necessary.

- Related to the last point is the fact that, in our approach, the MP reconstruction is algebraic (see Sec. IIE below), and does not involve differentiation of the field variables. This comes to be a great advantage in numerical applications, where differentiation often results in loss of numerical accuracy. In particular, in SF calculations it is necessary to resolve the MP near the particle with a great precision. The higher the order of the derivatives involved in constructing the $\mathrm{SF}$ are, the tougher become the resolution requirements. If the SF is to be constructed from the Weyl scalars (or from Moncrief's variables, as in [21]), this construction would necessitate taking three successive derivatives - two to reconstruct the MP, and a further one to obtain the force exerted by the MP. This has proven to be very demanding computationally [21]. In our approach, one need only take a single derivative of the numerical field variables.

- Yet another advantage of working with the Lorenz-gauge MP components as field variables, is that these behave more regularly near point particles than do Teukolsky's or Moncrief's variables. This has a simple manifestation when considering the multipole decomposition of the MP: The individual multipole modes of the Lorenz-gauge MP are continuous at the particle; only their first derivatives are discontinues there. The multipole modes of Teukolsky's or Moncrief's variables, on the other hand, are themselves discontinuous at the particle, and so are, in general, the modes of the MP in the RW gauge. Obviously, this better regularity of the Lorenz-gauge MP comes to be a great advantage when it comes to numerical implementation.

- The Lorenz-gauge perturbation equations take a fully hyperbolic form. (Compare with the situation in the RW or radiation gauges, where the set of perturbation equations split into a subset of hyperbolic field equations, and a subset of elliptic equations that constrain the evolution.) This makes the Lorenz-gauge formulation especially convenient for numerical applications which are based on time-domain evolution. The supplementary gauge conditions indeed take the form of elliptic "constraint" equations, but these can be made to hold automatically. We shall discuss this point in Sec. II.

- The Lorenz gauge condition does not interfere with the local isotropic symmetry of point particle singularities. This makes it well suited - in gravitational perturbation theory as in electromagnetism - for study of the local field near such particles. Other gauge choices (the RW and radiation gauges are examples) may artificially distort this symmetry, requiring a more cautious treatment.

- The use of (generalized) "harmonic coordinates", closely related to the choice of Lorenz gauge in a perturbative context, has lately been a popular trend in Numerical Relativity (e.g., [22]). It has been partially responsible for the significant progress made recently in fully non-linear simulations of binary black hole mergers [23]. There is still much to understand about the underlying mathematics that makes this formulation so successful. The simpler realm of perturbation theory can provide a good test bed for these ideas, and help gaining insight into some of the mathematical features of hyperbolic formulations.

The obvious down side of the Lorenz-gauge formulation is that one can no longer benefit from the kind of algebraic simplicity that has made the RW/radiation gauges so popular. There is no known way to fully decouple between the various tensorial components of the Lorenz-gauge MP, and one has to treat the perturbation equations as a coupled set. This, seemingly, has discouraged the development of a Lorenz-gauge MP formulation in the past. Part of our aim here is to demonstrate that such formulation is tractable, and accessible to numerical implementation, despite the lack of algebraic simplicity.

This paper is structured as follows. In Sec. II we formulate MP theory in the Lorenz gauge. Specializing to the case of a point mass source in Schwarzschild geometry, we decompose the field equations (and the gauge conditions) into tensor harmonic multipoles, and obtain a set of 10 hyperbolic wave equations for each multipole mode (each $l, m)$. This set couples between the various tensorial components of the MP; however, the MP variables are chosen in such a way that the equations are decoupled at their principle parts. Using the supplementary gauge conditions we modify the original wave equations to incorporate a "constraint damping" scheme: A mechanism designed to guarantee that violations of the gauge conditions (due, e.g., to numerical errors) are damped automatically during numerical evolution. In Sec. III we implement the above formulation for the case of a source particle in a circular geodesic orbit around the black hole. We give analytic solutions for the monopole mode of the MP, and for all of its axially-symmetric, odd-parity modes. To solve for the rest of the modes, we present a numerical code, based on 
characteristic time-evolution in $1+1$ dimensions. Section IV presents a series of validation tests for our code. In particular, we use our Lorenz-gauge solutions to extract the fluxes of energy (in each of the multipole modes) radiated to infinity in gravitational waves, and compare them with the values given in the literature. Finally, in Sec. V, we discuss the applicability of our approach to more general orbits in Schwarzschild, and to orbits around Kerr black holes.

\section{FORMULATION}

\section{A. Linearized Einstein equations in the Lorenz gauge}

Let $g_{\mu \nu}$ be the metric in a given "background" spacetime, which we assume to be Ricci-flat [i.e., $R_{\alpha \beta}\left(g_{\mu \nu}\right)=0$ ]. Let then $h_{\mu \nu}$ represent a small gravitational perturbation away from $g_{\mu \nu}$, produced by a given energy-momentum distribution $T_{\alpha \beta}$. Linearization of the Einstein equations, $G_{\alpha \beta}\left(g_{\mu \nu}+h_{\mu \nu}\right)=8 \pi T_{\alpha \beta}$, in the perturbation $h_{\mu \nu}$ about the background $g_{\mu \nu}$ yields the general form [11]

$$
\square h_{\alpha \beta}-g_{\alpha \beta} \square h+h_{; \alpha \beta}+2 R^{\mu}{ }_{\alpha}{ }^{\nu}{ }_{\beta} h_{\mu \nu}-h_{\alpha \mu^{; \mu} ; \beta}-h_{\beta \mu^{; \mu} ; \alpha}+g_{\alpha \beta} h_{\mu \nu}{ }^{; \mu \nu}=-16 \pi T_{\alpha \beta},
$$

where a semicolon denotes covariant differentiation in the background metric $g_{\mu \nu}, \square \equiv_{; \lambda^{; \lambda}}$ is the covariant D'Alambertian operator, $h \equiv g^{\mu \nu} h_{\mu \nu}$ is the trace of $h_{\mu \nu}$, and indices are raised and lowered using the background metric $g_{\mu \nu}$. Here, and throughout this paper, we follow the conventions of Ref. [24]; hence, the metric signature is $(-+++)$, the connection coefficients are $\Gamma_{\mu \nu}^{\lambda}=\frac{1}{2} g^{\lambda \sigma}\left(g_{\sigma \mu, \nu}+g_{\sigma \nu, \mu}-g_{\mu \nu, \sigma}\right)$, the Riemann tensor is $R_{\lambda \mu \nu}^{\alpha}=\Gamma_{\lambda \nu, \mu}^{\alpha}-\Gamma_{\lambda \mu, \nu}^{\alpha}+\Gamma_{\sigma \mu}^{\alpha} \Gamma_{\lambda \nu}^{\sigma}-\Gamma_{\sigma \nu}^{\alpha} \Gamma_{\lambda \mu}^{\sigma}$, the Ricci tensor and scalar are $R_{\alpha \beta}=R_{\alpha \mu \beta}^{\mu}$ and $R=R_{\alpha}^{\alpha}$, and the Einstein equations are $G_{\alpha \beta}=R_{\alpha \beta}-\frac{1}{2} g_{\alpha \beta} R=8 \pi T_{\alpha \beta}$. We shall use standard geometrized units, with $c=G=1$.

It is convenient to re-express the MP equations (1) in terms of the new variables

$$
\bar{h}_{\alpha \beta}=h_{\alpha \beta}-\frac{1}{2} g_{\alpha \beta} h .
$$

[Note $\bar{h}\left(\equiv \bar{h}_{\mu}{ }^{\mu}\right)=-h$; hence $\bar{h}_{\alpha \beta}$ is referred to as the "trace-reversed" MP.] Imposing the Lorenz gauge condition,

$$
\bar{h}_{\alpha \beta} ; \beta=0
$$

the MP equations reduce to the compact form

$$
\square \bar{h}_{\alpha \beta}(x)+2 R_{\alpha}^{\mu}{ }^{\nu}{ }_{\beta} \bar{h}_{\mu \nu}=-16 \pi T_{\alpha \beta} .
$$

This is a "linear, diagonal second order hyperbolic" system, which admits a well posed initial-value formulation on a spacelike Cauchy hypersurface (see, e.g., Theorem 10.1.2 of [25]). Furthermore, if the gauge conditions (3) are satisfied on the initial Cauchy surface, then they are guaranteed to hold everywhere [assuming that Eqs. (4) are satisfied everywhere, and that $\left.T_{\alpha \beta}{ }^{; \beta}=0\right] .^{2}$

The main motivation for this work comes from problems where the source of perturbation can be regarded as a "point particle", with a definite trajectory defined on the background metric. (See [1] for a discussion of how the notions of a "point particle" and a "trajectory" can be made sense of in a general-relativistic context.) In this case, the source term in the MP equations takes the form

$$
T_{\alpha \beta}\left(x^{\mu}\right)=\mu \int_{-\infty}^{\infty}(-g)^{-1 / 2} \delta^{4}\left[x^{\mu}-x_{\mathrm{p}}^{\mu}(\tau)\right] u_{\alpha} u_{\beta} d \tau \quad \text { (particle case) }
$$

where $\mu$ is the mass of the particle, $g$ is the determinant of $g_{\mu \nu}, x_{\mathrm{p}}^{\mu}(\tau)$ denotes the particle's worldline (parametrized by proper time $\tau$ ), and $u^{\alpha} \equiv d x_{\mathrm{p}}^{\alpha} / d \tau$ is a tangent 4 -velocity defined along the worldline.

\footnotetext{
${ }^{2}$ The conditions (3) do not fully specify the gauge: There is a residual gauge freedom within the family of Lorenz gauges, $h_{\alpha \beta} \rightarrow h_{\alpha \beta}+\xi_{\alpha ; \beta}+\xi_{\beta ; \alpha}$, with any $\xi^{\mu}$ satisfying $\square \xi^{\mu}=0$. It is easy to verify that the form of both (3) and (4) is invariant under such gauge transformations.
} 


\section{B. Multipole decomposition}

In the rest of this work we restrict our discussion to perturbations of the Schwarzschild black hole spacetime. The line element in the background geometry is then given by

$$
d s^{2}=-f d t^{2}+f^{-1} d r^{2}+r^{2}\left(d \theta^{2}+\sin ^{2} \theta d \varphi^{2}\right),
$$

where $f \equiv 1-2 M / r, M$ is the mass of the black hole, the event horizon is at $f(r)=0$, and $t, r, \theta, \varphi$ are the standard Schwarzschild coordinates, which we adopt throughout this paper. We shall proceed by decomposing the MP into multipole harmonics. This will be achieved by projecting $\bar{h}_{\alpha \beta}$ onto a basis of 2 nd-rank tensor harmonics, defined, in the background Schwarzschild geometry, on 2-spheres $t, r=$ const [26]. The spherical symmetry of the background geometry will guarantee that the individual multipole harmonics ("l, $m$ modes") are eigenvectors of the wave operator in Eq. (4), and hence evolve independently. However, the ten tensorial components of (each $l, m$ mode of) the perturbation will generally remain coupled.

We will adopt here the basis of tensor harmonics defined in Appendix A, which represents a slightly modified version of the one introduced by Zerilli [10]. We denote our tensor harmonics by $Y_{\alpha \beta}^{(i) l m}(\theta, \varphi ; r)$, where $l$ and $m$ are the multipole and azimuthal numbers, respectively, $i=1, \ldots, 10$ labels the ten elements of the tensorial basis, ${ }^{3}$ and $\alpha, \beta$ are tensorial indices. ${ }^{4}$ The harmonics $Y_{\alpha \beta}^{(i) l m}$ depend only on the Schwarzschild coordinates $\theta$ and $\varphi$, except for simple multiplicative powers of $r$ and $f(r)$ that are included in their definition (see Appendix A) - the former in the interest of dimensional balance, and the latter for regulating the behavior at the event horizon. With this definition, the noncoordinate-basis components $Y_{\hat{\alpha} \hat{\beta}}^{(i) l m} \equiv\left(g^{\alpha \alpha} g^{\beta \beta}\right)^{1 / 2} Y_{\alpha \beta}^{(i) l m}$ (no summation over repeated indices) are all dimensionless, and become $r$-independent at the limit $M / r \rightarrow 0$. Also, these harmonics are regular at the event horizon $[f(r)=0]$, in the sense that they attain finite (generally nonzero) values there when expressed in regular, "horizon penetrating" coordinates (like the Kruskal coordinates).

The $Y_{\alpha \beta}^{(i) l m}$, s constitute an orthonormal set, in the sense that

$$
\int d \Omega \eta^{\alpha \mu} \eta^{\beta \nu}\left[Y_{\mu \nu}^{(i) l m}\right]^{*} Y_{\alpha \beta}^{(j) l^{\prime} m^{\prime}}=\delta_{i j} \delta_{l l^{\prime}} \delta_{m m^{\prime}}
$$

(for any $i, j=1, \ldots, 10$ ), where $\eta^{\alpha \mu} \equiv \operatorname{diag}\left(1, f, r^{-2}, r^{-2} \sin ^{-2} \theta\right.$ ), an asterisk denotes complex conjugation, and the integration is carried over a 2 -sphere of constant $r$ and $t$. The seven harmonics $i=1, \ldots, 7$ constitute a basis for all covariant 2nd-rank symmetric tensors of even parity, while the remaining three harmonics $i=8,9,10$ span all such tensors which are of odd parity. Any covariant 2nd-rank symmetric tensor $t_{\alpha \beta}$ can hence be expanded as $t_{\alpha \beta}=$ $\sum_{l, m} \sum_{i=1}^{10} t^{(i) l m}(r, t) Y_{\alpha \beta}^{(i) l m}$, where the time-radial coefficients are given by $t^{(i) l m}(r, t)=\int d \Omega t_{\alpha \beta} \eta^{\alpha \mu} \eta^{\beta \nu}\left[Y_{\mu \nu}^{(i) l m}\right]^{*}$.

We expand the trace-reversed metric perturbation in the above tensor harmonics as

$$
\bar{h}_{\alpha \beta}=\frac{\mu}{r} \sum_{l, m} \sum_{i=1}^{10} a^{(i) l} \bar{h}^{(i) l m}(r, t) Y_{\alpha \beta}^{(i) l m}(\theta, \varphi ; r) .
$$

The coefficients $a^{(i) l}$ are introduced for the purpose of simplifying the form of Eqs. (18) below, and are given by

$$
a^{(i) l}=\frac{1}{\sqrt{2}} \times \begin{cases}1, & i=1,2,3,6 \\ {[l(l+1)]^{-1 / 2},} & i=4,5,8,9 \\ {[\lambda l(l+1)]^{-1 / 2},} & i=7,10\end{cases}
$$

where

$$
\lambda \equiv(l+2)(l-1)
$$

\footnotetext{
${ }^{3}$ At $l=0$ and $l=1$ there are actually fewer than ten independent basis elements: There are only four independent elements at $l=0$, and eight independent elements at each of the three $l=1$ modes - see Sec. II D below for a more detailed discussion.

${ }^{4}$ To avoid confusion we note that, despite the similar notation, the $Y_{\alpha \beta}^{(i) l m}$, s adopted here differ from the basis used by one of the authors in [27].
} 
The dimensionless scalar fields $\bar{h}^{(i) l m}(r, t)$ will serve as integration variables for the numerical time-evolution of the mode-decomposed perturbation equations. For that reason, the above construction is careful in making sure that these fields are well behaved (i.e., attain finite, generally nonzero values) both at spacial infinity and along the event horizon. The factor $1 / r$ in front of the expansion (8) was inserted to guarantee $\bar{h}^{(i) l m} \propto$ const as $r \rightarrow \infty$. The $\bar{h}^{(i) l m}$ 's are guaranteed to be regular at the horizon since both the physical perturbation $\bar{h}_{\alpha \beta}$ and the harmonics $Y_{\alpha \beta}^{(i) l m}$ are regular there.

In a similar manner, we expand the energy-momentum tensor as

$$
T_{\alpha \beta}=\sum_{l, m} \sum_{i=1}^{10} T^{(i) l m}(r, t) Y_{\alpha \beta}^{(i) l m}(\theta, \varphi ; r),
$$

with the time-radial coefficients given by

$$
T^{(i) l m}(r, t)=\int d \Omega \eta^{\alpha \mu} \eta^{\beta \nu}\left[Y_{\mu \nu}^{(i) l m}\right]^{*} T_{\alpha \beta} .
$$

For the point particle source with energy-momentum given in Eq. (5), this becomes

$$
T^{(i) l m}\left(r ; x_{\mathrm{p}}\right)=\frac{\mu}{u^{t} r_{\mathrm{p}}^{2}} u_{\alpha} u_{\beta} \eta^{\alpha \mu}\left(x_{\mathrm{p}}\right) \eta^{\beta \nu}\left(x_{\mathrm{p}}\right) Y_{\mu \nu}^{(i) l m *}\left(\Omega_{\mathrm{p}}\right) \delta\left(r-r_{\mathrm{p}}\right) \quad \text { (particle case) },
$$

where, recall, $x_{\mathrm{p}}(\tau)$ denotes the particle's trajectory, and $u^{\alpha}$ its 4 -velocity.

We now wish to obtain equations for the various fields $\bar{h}^{(i) l m}(r, t)$ (numbering ten, for each given $l, m$ ), that are fully separated with respect to $l, m$, and are uncoupled with respect to $i$ at their principle part. This is achieved by first substituting both expansions (8) and (11) into the field equations (4), and then constructing certain combinations of the resulting equations. For example, to obtain an equation for $\bar{h}^{(1) l m}$, one has to add the $t t$ component of Eq. (4) to the $r r$ component of that equation multiplied by $f^{2}$. For some of the other $\bar{h}^{(i) l m}$ 's one has to combine certain derivatives of the field equations. The full list of necessary combinations is given in Appendix B. This procedure yields a set of 10 equations, where the principle part of the $i$ 'th equation involves solely the $i^{\prime}$ th MP function, $\bar{h}^{(i) l m}$. One proceeds by showing that, in each of the 10 equations, the angular dependence on both sides is simply $\propto Y^{l m}$, and then using the orthogonality of the spherical harmonics to separate the equations into multipole modes.

To write the resulting separated equations in a convenient form, we introduce the standard 2-dimensional scalar-field wave operator (including centrifugal potential),

$$
\square_{\mathrm{sc}}^{2 d} \equiv \partial_{u v}+\frac{f}{4}\left[\frac{f^{\prime}}{r}+\frac{l(l+1)}{r^{2}}\right],
$$

where $f^{\prime} \equiv d f / d r=2 M / r^{2}$ and $v, u$ are the Eddington-Finkelstein null coordinates, defined by $v=t+r_{*}$ and $u=t-r_{*}$, with $d r_{*} / d r=f^{-1}$. (Throughout this paper it is to be understood that partial derivatives with respect to $v$ or $u$ are taken with fixed $u$ or $v$, respectively, while partial derivatives with respect to $t$ or $r$ are taken with fixed $r$ or $t$, respectively.) The separated field equations then take the form

$$
\square_{\mathrm{sc}}^{2 d} \bar{h}^{(i) l m}+\tilde{\mathcal{M}}_{(j)}^{(i) l} \bar{h}^{(j) l m}=4 \pi \mu^{-1}\left[r f / a^{(i)}\right] T^{(i) l m} \equiv S^{(i) l m} \quad(i=1, \ldots, 10) .
$$

The quantities $\tilde{\mathcal{M}}_{(j)}^{(i) l}$ are differential operators, of the first order at most, that couple between the various $\bar{h}^{(j)}$ 's (with same $l, m)$. The explicit form of the $\tilde{\mathcal{M}}_{(j)}^{(i) l}$, s can be found in Appendix C. As expected, one finds that the seven equations for the even-parity modes $\bar{h}^{(1, \ldots, 7)}$ decouple from the remaining 3 equations for the odd-parity modes $\bar{h}^{(8,9,10)}$ : We have $\tilde{\mathcal{M}}_{(j)}^{l(i)}=0$ for any $i=1, \ldots, 7$ with $j=8,9,10$, and for any $i=8,9,10$ with $j=1, \ldots, 7$. Further reduction of the system will be achieved in the next step, where we reemploy the gauge conditions.

\section{Gauge conditions and constraint damping}

The field equations (4) are supplemented by the gauge conditions $Z_{\alpha} \equiv \bar{h}_{\alpha \beta}^{; \beta}=0$ of Eq. (3). In a mode-decomposed form, these four conditions read

$$
H_{1}^{l m}(r, t) \equiv-\bar{h}_{, t}^{(1)}-\bar{h}_{, t}^{(3)}+f\left(\bar{h}_{, r}^{(2)}+\bar{h}^{(2)} / r-\bar{h}^{(4)} / r\right)=0
$$




$$
\begin{gathered}
H_{2}^{l m}(r, t) \equiv \bar{h}_{, t}^{(2)}-f\left(\bar{h}_{, r}^{(1)}-\bar{h}_{, r}^{(3)}\right)+(1-4 M / r) \bar{h}^{(3)} / r-(f / r)\left(\bar{h}^{(1)}-\bar{h}^{(5)}-2 f \bar{h}^{(6)}\right)=0, \\
H_{3}^{l m}(r, t) \equiv \bar{h}_{, t}^{(4)}-f\left(\bar{h}_{, r}^{(5)}+2 \bar{h}^{(5)} / r+l(l+1) \bar{h}^{(6)} / r-\bar{h}^{(7)} / r\right)=0, \\
H_{4}^{l m}(r, t) \equiv \bar{h}_{, t}^{(8)}-f\left(\bar{h}_{, r}^{(9)}+2 \bar{h}^{(9)} / r-\bar{h}^{(10)} / r\right)=0 .
\end{gathered}
$$

[To obtain these equations, insert the expansion (8) into the equations $Z_{t}=0, Z_{r}=0,\left(\sin \theta Z_{\theta}\right)_{, \theta}+\left(Z_{\varphi} / \sin \theta\right)_{,}=0$, and $Z_{\theta, \varphi}-Z_{\varphi, \theta}=0$, respectively; then show that the angular part in all cases is proportional to $Y^{l m}$ and use the orthogonality of the spherical harmonics to separate the equations.] These conditions, relating the various $\bar{h}^{(i)}$ 's and their first derivatives, are to supplement the separated field equations (15).

One now faces one of the standard problems of Numerical Relativity: the fact that the combined set of 10 field equations and 4 gauge conditions (or "constraints") is over-determined. Given initial data, the solutions are determined uniquely, in principle, by evolving the 10 field equations; how do we then make sure that the gauge conditions are satisfied as well during the evolution? In our case of Lorenz-gauge perturbations, and in the continuum limit, theory has it that if the gauge conditions are satisfied on the initial Cauchy surface, then the field equations will preserve them throughout the evolution. To see why this is true, simply take the divergence of Eq. (4), which, with the help of the contracted Bianchi identities and assuming Ricci flatness of the background geometry and $T_{\alpha \beta} ; \beta=0$, yields a simple homogeneous wave equation for the divergence of $\bar{h}_{\alpha \beta}: \square Z_{\alpha}=0$. Thus, in the continuum limit, if $\bar{h}_{\alpha \beta}$ satisfies a well-posed initial value problem and $Z_{\alpha}=0$ on the initial surface, then $Z_{\alpha}=0$ is guaranteed throughout the evolution. However, in actual numerical time-evolution implementations, gauge condition violations due to finite differentiating and/or roundoff errors can grow out of control even provided appropriate initial data. Moreover, in most cases it is practically impossible to devise initial data that satisfy the gauge conditions precisely, and at the same time are consistent with the field equations.

Gundlach et al. [28] recently proposed a general scheme for dealing with the above problem (in the wider context of fully non-linear Numerical Relativity), which employs the idea of constraint damping. Adapted to our problem of Lorenz-gauge perturbations, the idea would be to add to the linearized Einstein equations, Eqs. (4), a term of the form $-\kappa\left(t_{\alpha} Z_{\beta}+t_{\beta} Z_{\alpha}\right)$, where $\kappa$ is a positive constant and $t_{\alpha}$ is a future-directed timelike vector field. Obviously, the new system of $10+4$ equations is equivalent (in the continuum limit) to the original system. Also, the added term does not alter the principle part of the field equations and hence does not interfere with their neat hyperbolic form. However, the evolution equation for $Z_{\alpha}$ now becomes $\square Z_{\alpha}-\kappa\left(t_{\alpha} Z_{\beta}+t_{\beta} Z_{\alpha}\right)^{; \beta}=0$, which includes a damping term. One then expects that, under a range of circumstances [28], $Z_{\alpha}$ would "automatically" damp to zero during the evolution (with a timescale set by $\kappa$, if $t_{\alpha}$ is taken to be of unit length).

Here we will be inspired by the above scheme, but will allow ourselves an amount of freedom in executing it: We will seek to add terms $\propto Z_{\alpha}$ to our field equations, that would assure efficient damping of the constraints, and at the same time would lead to simplification in the final form of the decoupled field equations (putting in mind simple numerical implementation). The ultimate "justification" for the specific form selected for the added terms would come from numerical experiments with circular orbits, described in the following Sections.

We take $t_{\alpha}=-\delta_{\alpha}^{t}$, and add to the field equations (4) a term $\kappa\left(\delta_{\alpha}^{t} \tilde{Z}_{\beta}+\delta_{\beta}^{t} \tilde{Z}_{\alpha}\right)$, where $\kappa=\kappa(r)=f^{\prime}=2 M / r^{2}$ and $\tilde{Z}_{\alpha}=\left(Z_{r}, 2 Z_{r}, Z_{\theta}, Z_{\varphi}\right)$ (Schwarzschild components). This choice simplifies the form of the 10 field equations, and leads to partial decoupling as we describe below. Our $\kappa$ is not constant, but is expected to suit its purpose as it varies slowly, on a scale of the background curvature, which is much larger than the typical scale for constraint violations in evolution of perturbations from a point particle. (As our numerical experiments demonstrate, the latter scale tends to relate to the radius of curvature associated with the particle - see, for example, Figs. 10 in the next section.) The seemingly odd form of $\tilde{Z}_{\alpha}$ (note it has $Z_{r}$ for its $t$ component) has been selected based on numerical experiments with circular orbits. It turns out (see Sec. IV B) to yield efficient damping of all 4 constraints $Z_{\alpha}=0$, but we shall not attempt here to explain that on theoretical ground.

At the level of the mode-decomposed equations (17), the addition of the above term to the field equations amounts to adding $\left(f^{\prime} / 2\right) H_{2}$ at $i=1,2$, adding $\left(f^{\prime} / 4\right) H_{3}$ at $i=4,5$, and adding $\left(f^{\prime} / 4\right) H_{4}$ at $i=8,9$. This brings the separated field equations to their final form:

$$
\square_{\mathrm{sc}}^{2 d} \bar{h}^{(i) l m}+\mathcal{M}_{(j)}^{(i) l} \bar{h}^{(j) l m}=S^{(i) l m} \quad(i=1, \ldots, 10)
$$

where the terms $\mathcal{M}_{(j)}^{(i)} \bar{h}^{(j)}$ are given explicitly (omitting the indices $l, m$ for brevity) by 


$$
\begin{gathered}
\mathcal{M}_{(j)}^{(1)} \bar{h}^{(j)}=\frac{1}{2} f f^{\prime} \bar{h}_{, r}^{(3)}+\frac{f}{2 r^{2}}(1-4 M / r)\left(\bar{h}^{(1)}-\bar{h}^{(5)}\right)-\frac{1}{2 r^{2}}\left[1-6 M / r+12(M / r)^{2}\right] \bar{h}^{(3)}+\frac{f^{2}}{2 r^{2}}(6 M / r-1) \bar{h}^{(6)}, \\
\mathcal{M}_{(j)}^{(2)} \bar{h}^{(j)}=\frac{1}{2} f f^{\prime} \bar{h}_{, r}^{(3)}+f^{\prime}\left(\bar{h}_{, v}^{(2)}-\bar{h}_{, v}^{(1)}\right)+\frac{f^{2}}{2 r^{2}}\left(\bar{h}^{(2)}-\bar{h}^{(4)}\right)+\frac{1}{2}\left(f^{\prime} / r\right)\left[(1-4 M / r) \bar{h}^{(3)}-f\left(\bar{h}^{(1)}-\bar{h}^{(5)}-2 f \bar{h}^{(6)}\right)\right],
\end{gathered}
$$

$$
\begin{gathered}
\mathcal{M}_{(j)}^{(3)} \bar{h}^{(j)}=\frac{1}{2} f f^{\prime} \bar{h}_{, r}^{(3)}+\frac{1}{2 r^{2}}\left[1-8 M / r+10(M / r)^{2}\right] \bar{h}^{(3)}-\frac{f^{2}}{2 r^{2}}\left[\bar{h}^{(1)}-\bar{h}^{(5)}-(1-4 M / r) \bar{h}^{(6)}\right] \\
\mathcal{M}_{(j)}^{(4)} \bar{h}^{(j)}=\frac{1}{2} f^{\prime}\left(\bar{h}_{, v}^{(4)}-\bar{h}_{, v}^{(5)}\right)-\frac{1}{2} l(l+1)\left(f / r^{2}\right) \bar{h}^{(2)}-\frac{1}{4} f^{\prime} f / r\left[3 \bar{h}^{(4)}+2 \bar{h}^{(5)}-\bar{h}^{(7)}+l(l+1) \bar{h}^{(6)}\right] \\
\mathcal{M}_{(j)}^{(5)} \bar{h}^{(j)}=\frac{f}{r^{2}}\left[(1-4.5 M / r) \bar{h}^{(5)}-\frac{1}{2} l(l+1)\left(\bar{h}^{(1)}-\bar{h}^{(3)}\right)+\frac{1}{2}(1-3 M / r)\left(l(l+1) \bar{h}^{(6)}-\bar{h}^{(7)}\right)\right] \\
\mathcal{M}_{(j)}^{(6)} \bar{h}^{(j)}=-\frac{f}{2 r^{2}}\left[\bar{h}^{(1)}-\bar{h}^{(5)}-(1-4 M / r)\left(f^{-1} \bar{h}^{(3)}+\bar{h}^{(6)}\right)\right] \\
\mathcal{M}_{(j)}^{(7)} \bar{h}^{(j)}=-\frac{f}{2 r^{2}}\left(\bar{h}^{(7)}+\lambda \bar{h}^{(5)}\right) \\
\mathcal{M}_{(j)}^{(8)} \bar{h}^{(j)}=\frac{1}{2} f^{\prime}\left(\bar{h}_{, v}^{(8)}-\bar{h}_{, v}^{(9)}\right)-\frac{1}{4} f^{\prime} f / r\left(3 \bar{h}^{(8)}+2 \bar{h}^{(9)}-\bar{h}^{(10)}\right) \\
\mathcal{M}_{(j)}^{(9)} \bar{h}^{(j)}=\frac{f}{r^{2}(1-4.5 M / r) \bar{h}^{(9)}-\frac{f}{2 r^{2}}(1-3 M / r) \bar{h}^{(10)}} \\
\mathcal{M}_{(j)}^{(10)} \bar{h}^{(j)}=-\frac{f}{2 r^{2}}\left(\bar{h}^{(10)}+\lambda \bar{h}^{(9)}\right) .
\end{gathered}
$$

Recall in these equations $f=1-2 M / r, f^{\prime}=2 M / r^{2}, \lambda=(l+2)(l-1), \partial_{r}$ is taken with fixed $t$, and $\partial_{v}$ is taken with fixed $u$.

\section{Hierarchical structure of the separated field equations}

Following the above manipulations, the five equations for the even parity modes $i=1,3,5,6,7$ no longer couple to the remaining two equations for $i=2$, 4 . Similarly, in the odd-parity subset, the two equations for $i=9,10$ no longer couple to the third equations, for $i=8$. One can then solve the set of field equations (17) in a hierarchical manner, starting with the 5 even-parity equations for $\bar{h}^{(1,3,5,6,7)}$ and the 2 odd-parity equations for $\bar{h}^{(9,10)}$, and then using the solutions as source terms in the equations for $\bar{h}^{(2,4)}$ (even parity) and $\bar{h}^{(8)}$ (odd parity). This simplification is achieved regardless of the form of the source term $T_{\alpha \beta}$. Note that the functions $\bar{h}^{(2,4,8)}$ are those constructing the MP components $\bar{h}_{t r}, \bar{h}_{t \theta}$ and $\bar{h}_{t \varphi}$ [see Eqs. (20) below], which are associated with the shift vector on the surface $t=$ const.

The multipole sum in Eq. (8) [and in Eq. (11)] contains the two modes $l=0,1$. At these modes there are fewer than ten independent tensor-harmonic basis elements. At $l=0$, the "vectorial" elements $Y^{(4,5,8,9)}$ and "tensorial" elements $Y^{(7,10)}$ vanish identically, and the monopole MP is then composed, in general, of only the four "scalar" elements $Y^{(1,2,3,6)}$ (which are all even-parity). The system of field equations (17) thus reduces, at $l=0$, to a hierarchical set of 3 coupled equations for $\bar{h}^{(1,3,6)}$, plus a single equation for $\bar{h}^{(2)}$. As to the dipole, $l=1$ mode: Here, the two "tensorial" elements $Y^{(7,10)}$ vanish identically, and one is left with a hierarchical set of $4+2$ equations for the even parity modes, and a second hierarchical set of $1+1$ equations for the odd parity modes.

Table I summarizes the hierarchical structure of our separated field equations, for the different values of $l$. 


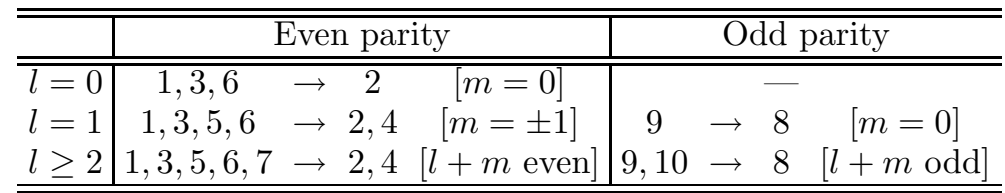

TABLE I. The hierarchical structure of the decoupled field equations (17) [numbers in this table refer to " $i$ " values of the tensorial-harmonic modes $\bar{h}^{(i)}$ ]: The full set of 10 equations first decouple into two subsets of 7 equations (even parity modes, $i=1, \ldots, 7$ ), and 3 equations (odd parity modes, $i=8,9,10$ ). Both even and odd sectors then further reduce into smaller subsets of equations (in a hierarchical sense): For modes with $l \geq 2$, the even-parity sector reduces to a subset of 5 equations, for $i=1,3,5,6,7$, whose solutions are then used as source terms in solving for $i=2,4$. Similarly, the odd-parity sector decouples into two (hierarchical) subsets of 2 and 1 equations. In the monopole and dipole cases the system is simpler: At $l=0$ the MP is purely even-parity, and one solves two (hierarchical) subsets with 3 and 1 equations. At $l=1$ one deals with $4+2$ equations in the even-parity sector, and $1+1$ equations in the odd-parity sector. When the source of the perturbation is an orbiting point particle, we can choose to work in a Schwarzschild coordinate system in which the orbit is confined to the equatorial plane $(\theta=\pi / 2)$. In this case, modes with even values of $l+m$ will be of pure even parity, while modes with odd values of $l+m$ will be of pure odd parity. The relevant $m$ modes that contribute to each of the entries of the table, in the case of a source particle in an equatorial orbit, are indicated in square brackets.

\section{E. Reconstruction of the metric perturbation}

Finally, it is useful to have at hand explicit formulas for reconstructing the various components of the original metric perturbation $h_{\alpha \beta}$, given the functions $\bar{h}^{(i) l m}(r, t)$. Using $h_{\alpha \beta}=\bar{h}_{\alpha \beta}-\frac{1}{2} g_{\alpha \beta} \bar{h}$, together with Eq. (8) and Appendix A, we find

$$
h_{\alpha \beta}=\frac{\mu}{2 r} \sum_{l=0}^{\infty} \sum_{m=-l}^{l} h_{\alpha \beta}^{l m},
$$

with

$$
\begin{aligned}
& h_{t t}^{l m}=\left(\bar{h}^{(1)}+f \bar{h}^{(6)}\right) Y^{l m}, \\
& h_{t r}^{l m}=f^{-1} \bar{h}^{(2)} Y^{l m}, \\
& h_{r r}^{l m}=f^{-2}\left(\bar{h}^{(1)}-f \bar{h}^{(6)}\right) Y^{l m}, \\
& h_{t \theta}^{l m}=r\left(\bar{h}^{(4)} Y_{\mathrm{V} 1}^{l m}+\bar{h}^{(8)} Y_{\mathrm{V} 2}^{l m}\right), \\
& h_{t \varphi}^{l m}=r \sin \theta\left(\bar{h}^{(4)} Y_{\mathrm{V} 2}^{l m}-\bar{h}^{(8)} Y_{\mathrm{V} 1}^{l m}\right), \\
& h_{r \theta}^{l m}=r f^{-1}\left(\bar{h}^{(5)} Y_{\mathrm{V} 1}^{l m}+\bar{h}^{(9)} Y_{\mathrm{V} 2}^{l m}\right), \\
& h_{r \varphi}^{l m}=i r f^{-1} \sin \theta\left(\bar{h}^{(5)} Y_{\mathrm{V} 2}^{l m}-\bar{h}^{(9)} Y_{\mathrm{V} 1}^{l m}\right), \\
& h_{\theta \theta}^{l m}=r^{2}\left(f^{-1} \bar{h}^{(3)} Y^{l m}+\bar{h}^{(7)} Y_{\mathrm{T} 1}^{l m}+\bar{h}^{(10)} Y_{\mathrm{T} 2}^{l m}\right), \\
& h_{\theta \varphi}^{l m}=i r^{2} \sin \theta\left(\bar{h}^{(7)} Y_{\mathrm{T} 2}^{l m}-\bar{h}^{(10)} Y_{\mathrm{T} 1}^{l m}\right), \\
& h_{\varphi \varphi}^{l m}=r^{2} \sin ^{2} \theta\left(f^{-1} \bar{h}^{(3)} Y^{l m}-\bar{h}^{(7)} Y_{\mathrm{T} 1}^{l m}-\bar{h}^{(10)} Y_{\mathrm{T} 2}^{l m}\right),
\end{aligned}
$$

where we have omitted the indices $l m$ from $\bar{h}^{(i) l m}$ for brevity, and where $Y_{\mathrm{V} 1}^{l m}, Y_{\mathrm{V} 2}^{l m}, Y_{\mathrm{T} 1}^{l m}$, and $Y_{\mathrm{T} 2}^{l m}$ are angular functions constructed from the spherical harmonics through

$$
\begin{aligned}
Y_{\mathrm{V} 1}^{l m} & \equiv \frac{1}{l(l+1)} Y_{, \theta}^{l m}, \\
Y_{\mathrm{V} 2}^{l m} & \equiv \frac{1}{l(l+1)} \sin ^{-1} \theta Y_{, \varphi}^{l m}, \\
Y_{\mathrm{T} 1}^{l m} & \equiv \frac{1}{\lambda l(l+1)}\left[\sin \theta\left(\sin ^{-1} \theta Y_{, \theta}^{l m}\right)_{, \theta}-\sin ^{-2} \theta Y_{, \varphi \varphi}^{l m}\right], \\
Y_{\mathrm{T} 2}^{l m} & \equiv \frac{2}{\lambda l(l+1)}\left(\sin ^{-1} \theta Y_{, \varphi}^{l m}\right)_{, \theta} .
\end{aligned}
$$


Note Eq. (19) gives the MP itself, not its trace-reversed counterpart. In applying Eqs. (20) to $l=0,1$, recall $Y_{\mathrm{V} 1, \mathrm{~V} 2}^{l=0}=Y_{\mathrm{T} 1, \mathrm{~T} 2}^{l=0} \equiv 0$ and $Y_{\mathrm{T} 1, \mathrm{~T} 2}^{l=1} \equiv 0$. Finally, note that the trace of the MP is simply given by

$$
h \equiv g^{\alpha \beta} h_{\alpha \beta}=(\mu / r) \sum_{l m}\left(f^{-1} \bar{h}^{(3)}-\bar{h}^{(6)}\right) Y^{l m} .
$$

This implies that the function $\bar{h}^{(3)}$ must vanish at the event horizon (where $f=0$ ), since the trace $h$ must be regular at the horizon, and the functions $\bar{h}^{(3)}$ and $\bar{h}^{(6)}$ are both finite there by construction.

The various fields $h_{\alpha \beta}^{l m}$ are complex quantities. However, the sum $\sum_{m} h_{\alpha \beta}^{l m}$ is guaranteed to be real. From the definition of the harmonics $Y^{(i) l m}$ in App. A, and the decoupled field equations (17), one readily verifies the symmetry relation

$$
\left(\bar{h}^{(i)} Y^{(i) l m}\right)_{m \rightarrow-m}=\left(\bar{h}^{(i)} Y^{(i) l m}\right)^{*}
$$

(namely, the product $\bar{h}^{(i)} Y^{(i) l m}$ is invariant under simultaneous sign-reversal of $m$ and complex conjugation), which is valid for each $i$ and any $l$. Hence, the sum over modes $m$ in the reconstruction formula (19) can be written in the form

$$
\sum_{m=-l}^{l} h_{\alpha \beta}^{l m}=h_{\alpha \beta}^{l, m=0}+2 \sum_{m=1}^{l} \operatorname{Re}\left(h_{\alpha \beta}^{l m}\right),
$$

which is manifestly real. In practice, this allows a more economic implementation of the reconstruction scheme: One need only compute the $m \geq 0$ modes to reconstruct the full MP.

\section{IMPLEMENTATION: CASE OF A PARTICLE IN A CIRCULAR GEODESIC ORBIT}

\section{A. Setup}

Consider a pointlike particle of mass $\mu$, in a circular orbit around a Schwarzschild black hole with mass $M \gg \mu$. Neglecting SF effects, the particle traces a geodesic $x^{\alpha}=x_{\mathrm{p}}^{\alpha}(\tau)$, with four velocity $u^{\alpha} \equiv d x_{\mathrm{p}}^{\alpha} / d \tau$. In what follows we work in a Schwarzschild coordinate system $t, r, \theta, \varphi$ at which the orbit is confined to the equatorial plane:

$$
x_{p}^{\alpha}(\tau)=\left[t(\tau), r_{0}=\text { const, } \theta_{0}=\pi / 2, \varphi(\tau)\right] .
$$

This geodesic is completely parametrized by the radius $r_{0}$, or, alternatively, by an "angular velocity"

$$
\omega \equiv d \varphi / d t=\sqrt{M / r_{0}^{3}}
$$

The circular geodesic can also be parameterized by the (conserved) specific energy, given by

$$
\mathcal{E} \equiv-u_{t}=f_{0}\left(1-3 M / r_{0}\right)^{-1 / 2},
$$

where $f_{0} \equiv 1-2 M / r_{0}$. The four velocity of the particle is then given (in Schwarzschild coordinates) by

$$
u^{\alpha}=\left(\mathcal{E} / f_{0}\right)[1,0,0, \omega] .
$$

Our goal here is to calculate the physical (stationary) MP associated with this orbiting particle, in the Lorenz gauge.

\section{B. Source terms}

From Eqs. (13) and (15) we obtain the source terms for our decoupled filed equations (17). They read

$$
S^{(i) l m}(r, t)=4 \pi \mathcal{E} \alpha^{(i)} \delta\left(r-r_{0}\right) \times\left\{\begin{array}{l}
Y^{l m *}(\pi / 2, \omega t), i=1-7 \text { (even), } \\
Y_{, \theta}^{l m *}(\pi / 2, \omega t), i=8-10 \text { (odd), }
\end{array}\right.
$$


where the coefficients $\alpha^{(i)}$ are given by

$$
\begin{aligned}
\alpha^{(1)} & =\alpha^{(3)}=f_{0}^{2} / r_{0}, \\
\alpha^{(2)} & =\alpha^{(5)}=\alpha^{(9)}=0, \\
\alpha^{(4)} & =2 i f_{0} m \omega, \\
\alpha^{(6)} & =r_{0} \omega^{2}, \\
\alpha^{(7)} & =r_{0} \omega^{2}\left[l(l+1)-2 m^{2}\right], \\
\alpha^{(8)} & =2 f_{0} \omega, \\
\alpha^{(10)} & =2 i m r_{0} \omega^{2} .
\end{aligned}
$$

Note the special case $m=0$ : For these axially-symmetric modes the field equations for both $\bar{h}^{(9)}$ and $\bar{h}^{(10)}$ are sourceless, and (since these two equations do not couple to any of the other $\bar{h}^{(i)}$ 's) one finds

$$
\bar{h}_{m=0}^{(9)}=\bar{h}_{m=0}^{(10)}=0 .
$$

The entire axially-symmetric odd-parity perturbation is then described by a single function $\bar{h}^{(8)}$, satisfying a closedform equation. Since, in the circular orbit case, $m=0$ modes are static, this equation is in fact an ordinary differential equation (ODE), and is readily solvable analytically. Below we construct the analytic solutions for these axially-symmetric odd-parity modes.

In the even-parity sector, both functions $\bar{h}^{(2)}$ and $\bar{h}^{(4)}$ have vanishing source terms at $m=0$. Inspecting the field equations in their form (15), with Eqs. (C1b) and (C1d), we observe that $\bar{h}^{(2)}$ and $\bar{h}^{(4)}$ couple not only to each other, but also to $\bar{h}^{(1)}$ and $\bar{h}^{(5)}$. However, since this coupling occurs through $t$ derivative terms, and since in our circular-orbit case $m=0$ modes are static, these coupling terms in fact vanish, and we find

$$
\bar{h}_{m=0}^{(2)}=\bar{h}_{m=0}^{(4)}=0 .
$$

Hence, the axially-symmetric even-parity part of the perturbation is described by the 5 functions $\bar{h}^{(1,3,5,6,7)}$, which satisfy a coupled set of ODEs.

\section{Analytic solutions for the axially-symmetric, odd-parity modes}

As we explained above, in the circular-orbit case, axially-symmetric $(m=0)$ odd-parity modes of the MP are constructed, at each $l$, from the single function $\bar{h}_{m=0}^{(8)}$, which is $t$-independent in this case. Denoting $\bar{h}_{m=0}^{(8)} \equiv \phi_{l}(r)$, the field equation for $\bar{h}^{(8)}$ [Eqs. (17) with (18h)] takes the form

$$
\phi_{l}^{\prime \prime}+V_{l}(r) \phi_{l}=-4 f^{-2} S_{m=0}^{(8)}=\beta_{l} \times \delta\left(r-r_{0}\right),
$$

where a prime denotes $d / d r$,

$$
V_{l}(r)=-f^{-1}(r)\left[\frac{l(l+1)}{r^{2}}-\frac{4 M}{r^{3}}\right]
$$

and the coefficient $\beta_{l}$ is given by

$$
\begin{aligned}
\beta_{l} & =-32 \pi f_{0}^{-1} \mathcal{E} \omega Y_{, \theta}^{l, m=0}(\theta=\pi / 2) \\
& = \begin{cases}16 f_{0}^{-1} \mathcal{E} \omega(-1)^{(l-1) / 2}[\pi(2 l+1)]^{1 / 2} l ! ! /(l-1) ! !, & l \text { odd }, \\
0, & l \text { even. }\end{cases}
\end{aligned}
$$

Hence, we have $\phi_{l}(r) \equiv 0$ for all modes with even values of $l$, and we need only consider modes with odd $l$ values.

Two independent homogeneous solutions to Eq. (33) are given, for $l \geq 2$ (we will discuss the mode $l=1$ separately below), by

$$
\begin{aligned}
& \phi_{l \geq 2}^{\mathrm{EH}}(r)=\frac{x}{1+x} \sum_{n=0}^{l+1} a_{n}^{l} x^{n}, \\
& \phi_{l \geq 2}^{\infty}(r)=\phi_{l}^{\mathrm{EH}} \ln f+\frac{1}{1+x} \sum_{n=0}^{l+1} b_{n}^{l} x^{n},
\end{aligned}
$$


where

$$
x \equiv r /(2 M)-1
$$

and the coefficients read

$$
a_{n}^{l}=\frac{l(l+1)(l+n-1) !}{(l-n+1) !(n+1) ! n !}, \quad b_{n}^{l}=\sum_{k=0}^{l-n+1}(-1)^{k} \frac{a_{n+k}^{l}}{k+1} .
$$

These solutions have the following asymptotic behavior at the horizon $(r \rightarrow 2 M, x \rightarrow 0, f \rightarrow 0)$ and at infinity $(r, x \rightarrow \infty)$ :

$$
\begin{gathered}
\phi_{l \geq 2}^{\mathrm{EH}} \propto \begin{cases}f, & r \rightarrow 2 M, \\
r^{l+1}, & r \rightarrow \infty,\end{cases} \\
\phi_{l \geq 2}^{\infty} \propto \begin{cases}f \ln f, & r \rightarrow 2 M, \\
r^{-l}, & r \rightarrow \infty .\end{cases}
\end{gathered}
$$

The solution $\phi_{l}^{\mathrm{EH}}$ is regular (analytic) at the horizon but diverges at $r \rightarrow \infty$, whereas the solution $\phi_{l}^{\infty}$ is regular at $r \rightarrow \infty$ but irregular at the horizon (it vanishes there, but it is non-differentiable). Matching these solutions at the particle's location $\left(r=r_{0}\right)$, we hence construct a unique regular, continuous solution for the inhomogeneous Eq. (33):

$$
\phi_{l \geq 2}=-2 M \lambda \beta_{l} \times \begin{cases}\phi_{l}^{\mathrm{EH}}(r) \phi_{l}^{\infty}\left(r_{0}\right), & r \leq r_{0}, \\ \phi_{l}^{\infty}(r) \phi_{l}^{\mathrm{EH}}\left(r_{0}\right), & r \geq r_{0},\end{cases}
$$

where, recall, $\lambda=(l+2)(l-1)$. In obtaining this solution we have used the fact that the Wronskian $W \equiv \phi^{\mathrm{EH}}\left[\phi^{\infty}\right]^{\prime}-$ $\left[\phi^{\mathrm{EH}}\right]^{\prime} \phi^{\infty}$ must be constant [since the ODE (33) contains no $\phi_{l}^{\prime}$ term]. Evaluating it at $x=0$ one then easily obtains $W=-b_{0}^{l} /(2 M)=-(2 M \lambda)^{-1}$.

At $l=1$, the function $\phi_{l}^{\infty}$ of Eq. (36) fails to be a solution of the homogeneous part of Eq. (33) (although $\phi_{l=1}^{\mathrm{EH}}$ still is a solution). Instead, the general homogeneous solution takes the simple form

$$
\phi_{l=1}=a r^{2}+b / r
$$

where $a$ and $b$ are constants. [Note that for $l=1$ the effective potential in Eq. (33) reduces to simply $V(r)=-2 / r^{2}$.] The coefficients $a$ and $b$ are determined uniquely by requiring regularity at the horizon ${ }^{5}$ and at infinity, and imposing continuity at $r=r_{0}$, along with a 'jump' condition for the derivative there: $\left[\phi_{l=1}^{\prime}\right]_{r_{0}}=\beta_{l=1}$. This yields

$$
\phi_{l=1}=-\frac{1}{3} r_{0} \beta_{l=1} \times \begin{cases}\left(r / r_{0}\right)^{2}, & r \leq r_{0}, \\ \left(r_{0} / r\right), & r \geq r_{0},\end{cases}
$$

with $\beta_{l=1}=16 \sqrt{3 \pi} f_{0}^{-1} \mathcal{E} \omega$.

We can now write down explicitly the solution for the axially-symmetric, odd-parity part of the MP $h_{\alpha \beta}$ itself. From the reconstruction equations (19) [with Eqs. (20)], recalling $Y_{\mathrm{V} 2}^{l m}=0$ at $m=0$, we find that the only non-vanishing axially-symmetric, odd-parity components are $h_{t \varphi}=h_{\varphi t}$, given by

$$
\begin{aligned}
h_{t \varphi}[m=0, \text { odd }] & =-\frac{\mu}{2} \sum_{\text {odd } l} \sin \theta \bar{h}_{m=0}^{(8)} Y_{\mathrm{V} 1}^{l, m=0} \\
& =-\sum_{\text {odd } l} \frac{\mu}{2 l(l+1)} \phi_{l}(r) \sin \theta Y_{, \theta}^{l, m=0}(\theta) .
\end{aligned}
$$

\footnotetext{
${ }^{5}$ There is a subtlety here: The homogeneous solution $\propto 1 / r$ is regular everywhere for any finite $M$ and $r_{0}$. However, we do wish to require that the solution remains regular even at the limit $M \rightarrow 0$ (taken with fixed $r_{0}$, which is mathematically equivalent to the limit $r_{0} \rightarrow \infty$, taken with fixed $M$ ). This excludes the solution $\propto 1 / r$ at $r<r_{0}$, as it grows unboundedly at the horizon in that limit.
} 
The lowest multipole contribution to the sum in Eq. (44) is a "conservative" piece coming from the dipole mode, $l=1$. It reads

$$
\begin{aligned}
h_{t \varphi}[m=0, l=1] & =-\frac{1}{4} \mu \phi_{l=1} \sin \theta Y_{, \theta}^{l=1, m=0} \\
& =-2 \mu f_{0}^{-1} \mathcal{E} \omega r_{0} \sin ^{2} \theta \times \begin{cases}\left(r / r_{0}\right)^{2}, & r \leq r_{0}, \\
\left(r_{0} / r\right), & r \geq r_{0} .\end{cases}
\end{aligned}
$$

This agrees with the odd-parity dipole solution first obtained by Zerilli [11], which, as pointed out recently by Detweiler and Poisson [29], is a Lorenz-gauge solution. [In comparing our solution (45) with Eq. (4.1) of [29], note $f_{0}^{-1} \mathcal{E} \omega r_{0}^{2}=\left[M r_{0} /\left(1-3 M / r_{0}\right)\right]^{1 / 2}$ is the particle's specific angular momentum, denoted $\tilde{L}$ in [29].] This piece of the MP describes the shift in the angular momentum content of the perturbation across the surface $r=r_{0}$.

\section{Analytic solution for the monopole $(l=0)$ mode}

The lowest multipole contribution to the MP comes from the monopole, $l=m=0$ mode. This "conservative" piece of the MP (which is purely even parity) describes the shift in the mass parameter of the perturbation across the surface $r=r_{0}$. As was the case with the $m=0$, odd parity modes considered above, at $l=0$, too, the field equations (17) simplify enough that one can obtain the solution analytically, with moderate effort. This solution was derived recently by Detweiler and Poisson [29]. ${ }^{6}$ For the sake of completeness, and since Detweiler and Poisson do not actually write down their solution explicitly (as they are interested mainly in the SF exerted by the monopole, not the monopole MP itself), we bring this solution here.

At $r \leq r_{0}$, the non-vanishing components of the Lorenz-gauge monopole perturbation are given by

$$
\begin{gathered}
h_{t t}^{l=0}\left(r \leq r_{0}\right)=-\frac{A f M}{r^{3}} P(r), \\
h_{r r}^{l=0}\left(r \leq r_{0}\right)=\frac{A}{r^{3} f} Q(r), \\
h_{\theta \theta}^{l=0}\left(r \leq r_{0}\right)=(\sin \theta)^{-2} h_{\varphi \varphi}^{l=0}\left(r \leq r_{0}\right)=\operatorname{AfP}(r),
\end{gathered}
$$

where

$$
\begin{gathered}
A=\frac{2 \mu \mathcal{E}}{3 M r_{0} f_{0}}\left[M-\left(r_{0}-3 M\right) \ln f_{0}\right], \\
P(r)=r^{2}+2 M r+4 M^{2}, \quad Q(r)=r^{3}-M r^{2}-2 M^{2} r+12 M^{3} .
\end{gathered}
$$

At $r \geq r_{0}$, the solutions read

$$
\begin{aligned}
& h_{t t}^{l=0}\left(r \geq r_{0}\right)= \frac{2 \mu \mathcal{E}}{3 r^{4} r_{0} f_{0}}\left\{3 r^{3}\left(r_{0}-r\right)+M^{2}\left(r_{0}^{2}-12 M r_{0}+8 M^{2}\right)+\right. \\
&\left.\left(r_{0}-3 M\right)\left[-r M(r+4 M)+r P(r) f \ln f+8 M^{3} \ln \left(r_{0} / r\right)\right]\right\}, \\
& h_{r r}^{l=0}\left(r \geq r_{0}\right)=- \frac{2 \mu \mathcal{E}}{3 r^{4} r_{0} f_{0} f^{2}}\left\{-r^{3} r_{0}-2 M r\left(r_{0}^{2}-6 M r_{0}-10 M^{2}\right)+3 M^{2}\left(r_{0}^{2}-12 M r_{0}+8 M^{2}\right)+\right. \\
&\left.\left(r_{0}-3 M\right)\left[5 M r^{2}+(r / M) Q(r) f \ln f-8 M^{2}(2 r-3 M) \ln \left(r_{0} / r\right)\right]\right\},
\end{aligned}
$$

\footnotetext{
${ }^{6}$ In fact, Detweiler and Poisson did not obtain their solution by directly tackling the Lorenz-gauge equations. Rather, they started with the $l=0$ solution derived by Zerilli [11] in a non-Lorenz gauge, and then, essentially, solved the gauge transformation equations that take Zerilli's solution to the Lorenz gauge.
} 


$$
\begin{aligned}
h_{\theta \theta}^{l=0}\left(r \geq r_{0}\right)= & (\sin \theta)^{-2} h_{\varphi \varphi}^{l=0}\left(r \geq r_{0}\right)=-\frac{2 \mu \mathcal{E}}{9 r r_{0} f_{0}}\left\{3 r_{0}^{2} M-80 M^{2} r_{0}+156 M^{3}+\right. \\
& \left.\left(r_{0}-3 M\right)\left[-3 r^{2}-12 M r+3(r / M) P(r) f \ln f+44 M^{2}+24 M^{2} \ln \left(r_{0} / r\right)\right]\right\} .
\end{aligned}
$$

It can be readily verified that these solutions match continuously at $r=r_{0}$. It can also be checked that they satisfy both the field equations (17) and the gauge conditions (16). For this, note that at $l=0$, in the circular orbit case, the only non-vanishing $\bar{h}^{(i)}$ 's are $\bar{h}^{(1,3,6)}$, and use the relations $\bar{h}_{l=0}^{(1)}=2 \sqrt{\pi} \mu^{-1} r\left(h_{t t}+f^{2} h_{r r}\right), \bar{h}_{l=0}^{(6)}=2 \sqrt{\pi} \mu^{-1} r f^{-1}\left(h_{t t}-f^{2} h_{r r}\right)$, and $\bar{h}_{l=0}^{(3)}=4 \sqrt{\pi} \mu^{-1}(f / r) h_{\theta \theta}$.

The above monopole solution is regular both at the event horizon and at infinity, taking the asymptotic forms

$$
\left.\begin{array}{c}
h_{t t}^{l=0}=-\frac{2}{3} A f+O\left(f^{2}\right) \\
f^{2} h_{r r}^{l=0}=\frac{2}{3} A f+O\left(f^{2}\right) \\
h_{\theta \theta}^{l=0}=12 M^{2} A f+O\left(f^{3}\right)
\end{array}\right\} \quad \text { as } r \rightarrow 2 M(f \rightarrow 0),
$$

Detweiler and Poisson show [29] that this is a unique Lorenz-gauge solution which is regular both at the event horizon and at infinity: Any gauge transformation of this solution within the class of Lorenz gauges would lead to irregular behavior at one (or both) of these asymptotic domains. ${ }^{7}$

Physically, the monopole perturbation of Eqs. (46) and (49) describes a shift in the Schwarzschild mass across $r=r_{0}$. This is most clearly evident from Zerilli's form of the monopole solution [10] (which differs from the above Lorenz-gauge solution only by a gauge transformation [29]), where it is easily seen that the geometry described by $g_{\alpha}+h_{\alpha \beta}^{l=0}$ is that of a Schwarzschild black hole with mass $M$ at $r<r_{0}$, and that of yet another Schwarzschild black hole, with mass $M+\mu \mathcal{E}$, at $r>r_{0}$.

The above Lorenz-gauge monopole solution is plotted in Fig. 1, for a sample of $r_{0}$ values.

\section{E. Numerical solutions for the rest of the modes}

For the modes considered so far (those with $m=0$ and $l=0,1,3,5, \ldots$ ) the field equations simplify enough to allow a fully analytic treatment (as least within the simplicity of the circular orbit case). To solve for the rest of the modes we will resort to numerical methods, employing the full machinery of the formalism developed in Sec. II. In the rest of this section we briefly describe our numerical method, and plot a sample of numerical solutions. In the next section we shall present various validation tests for our numerical code.

We need to integrate, numerically, the set of coupled field equations (17), with the source terms given in Eq. (29). Recall that for each given $l, m$, we are facing two sets of 7 (even parity) and 3 (odd parity) equations, which couple in the manner described in Table I (but recall that no coupling occurs at the principal parts of the equations). We choose to integrate these equations in the time domain, i.e., without introducing a Fourier decomposition. A frequency-domain analysis (of the sort employed many times in the past-see, e.g., [30]) is likely to be more efficient, numerically, for studying circular orbits. Our strong motivation in developing a time-domain evolution code stems from the fact that such a code is readily extensible to any type of orbit, with arbitrary eccentricity. A time-domain code can handle radial plunge trajectories as efficiently as it handles circular orbits. Frequency-domains codes, on the other hand, quickly loose their efficiency with growing eccentricity, as the number of Fourier modes one must sum over grow rapidly with eccentricity. Orbits with eccentricities greater than $\sim 0.7$ are essentially intractable with a frequency-domain treatment [31]. Another reason to opt for a time evolution approach, especially having in mind SF applications, is the following: A frequency domain analysis of the field equations requires careful implementation of boundary conditions, which becomes increasingly difficult with increasing $l$ values. The technical reason for this is explained, e.g., by Hughes in [32]. With a time domain evolution one can avoid this difficulty, either by expanding

\footnotetext{
${ }^{7}$ Note, however, the peculiar feature of the above solution, $h_{t t} \rightarrow \operatorname{const}\left[=-2 \mu \mathcal{E}\left(r_{0} f_{0}\right)^{-1}\right]$ at $r \rightarrow \infty$, which means that the perturbed metric, expressed in Schwarzschild coordinates, does not tend to the Minkowski metric at infinity. (Recall, however, that this peculiarity merely relates to the choice of gauge; the underlying perturbed geometry is, of course, asymptotically flat.)
} 

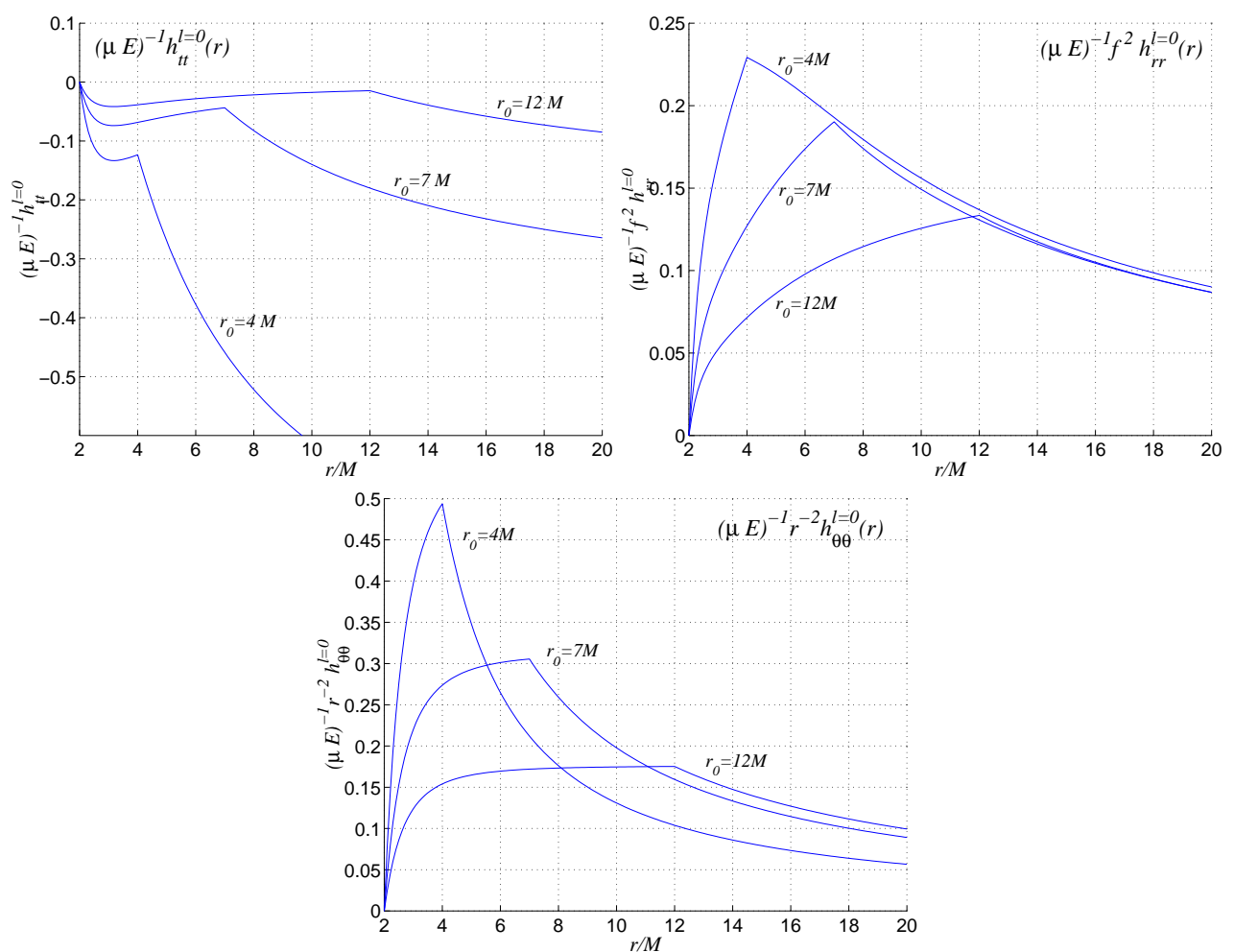

FIG. 1. The Lorenz-gauge monopole solution, Eqs. (46) and (49), plotted here for three values of the orbital radius, $r_{0}=4 M, 7 M, 12 M$. By construction (Detweiler and Poisson, Ref. [29]) this solution is continuous across the orbit, and well behaved both at the event horizon and at infinity. It is a unique Lorenz-gauge monopole solution with these properties. (The quantity $E$ is the specific energy parameter, denoted elsewhere in this paper by $\mathcal{E}$.)

the spacial boundaries of the numerical domain such as to dismiss any boundary effects, or by using characteristic evolution (as described below) which avoids boundaries altogether. Hence, a time domain approach should allow better accessibility to the higher $l$ modes.

A suitable numerical method, for time evolution of the field equations with a particle source represented by a delta function, was first presented by Lousto and Price [33]. It has since been implemented in a variety of a cases: A radially-falling scalar charge [34], a radially-falling mass particle [21,35], a mass particle in a circular orbit [36], and, lately, a mass particle in eccentric and parabolic orbits [37]. In the scalar field case [34] the source term in the decoupled field equations [the scalar-field equivalent of our Eqs. (17)] is simply proportional to a delta function $\delta\left[r-r_{\mathrm{p}}(\tau)\right]$. However, in the gravitational field case, all above works [21,35-37] employed the Regge-Wheeler-ZerilliMoncrief's formulation, where the source involves also terms proportional to $d \delta\left[r-r_{\mathrm{p}}(\tau)\right] / d r$. As a consequence, solutions to the Regge-Wheeler-Zerilli-Moncrief equations turn out discontinuous across the orbit, which, of course, complicates considerably the implementation of the above numerical method. One great advantage of working with the Lorenz-gauge MP, is that the source term in the field equations involves only a delta function, no derivatives thereof - as in the simple scalar case. The solutions are then continuous everywhere, and the implementation of the numerical integration scheme of Ref. [33] becomes must simpler.

Our numerical code is based on characteristic evolution and uses double-null coordinates $v \equiv t+r_{*}$ and $u \equiv t-r_{*}$ (like in [34], but unlike, e.g., in [37]). The numerical domain is a two dimensional fixed-step grid, as illustrated in Fig. 2. The evolution starts with characteristic data on $v=v_{0}$ and $u=u_{0}$, where we take $v_{0}=r_{*}\left(r_{0}\right)$ and $u_{0}=-r_{*}\left(r_{0}\right)$. That is, we take $t=0$ at the initial instance of the evolution [represented by the vortex $\left(v_{0}, u_{0}\right)$ ], and take the two initial null surfaces to be the ingoing and outgoing light rays emanating from the particle at that instance. The location of the event horizon on this grid is approximated by a large value of $u$ (with constant $v$ ), while large value of $v$ (with constant $u$ ) approximates the location of null infinity. The trajectory of the particle is represented by the vertical line $v-u=2 r_{*}\left(r_{0}\right)$, connecting the lower- and upper-most vertices of the grid. Since our numerical evolution variables are continuous at the trajectory, there are no complication involved in taking the trajectory to cut through grid points, as we do here; in fact, we found this setup most convenient.

The numerical evolution is based on the finite difference scheme developed in [33] — as simplified to the case where the source term contains no derivatives of a delta function, and generalized to deal with a set of simultaneous equations. 


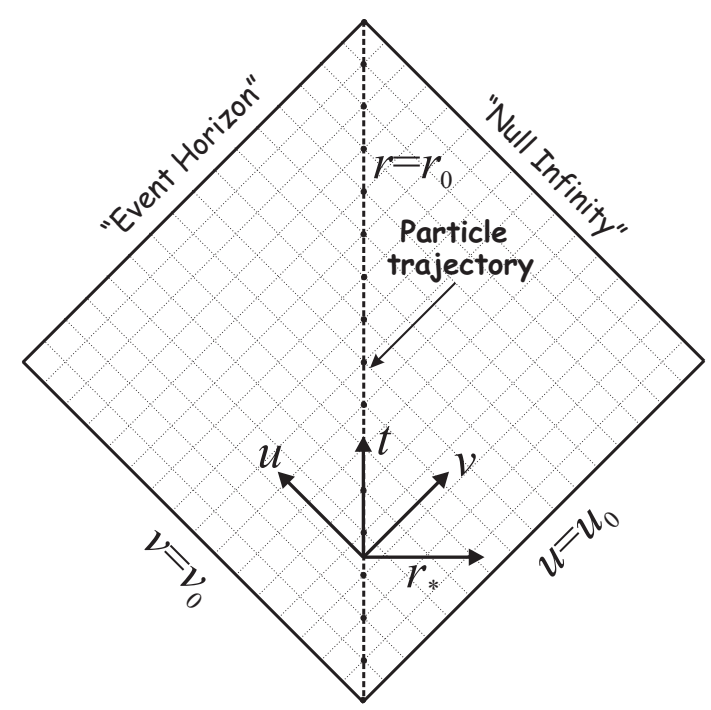

FIG. 2. Numerical grid for characteristic evolution of the field equations. See the text for description.

Since the scheme has been described in detail elsewhere, we shall not elaborate on it here, but rather refer the reader to [33] or [35]. The finite difference algorithm is second order convergent. The value of the solution at a grid point with coordinates $\left(v_{i}, u_{i}\right)$ is approximated, within an error quadratic in the step size, based on the values at the three grid points $\left(v_{i-1}, u_{i}\right),\left(v_{i}, u_{i-1}\right)$, and $\left(v_{i-1}, u_{i-1}\right)$, which have been solved for in previous steps. For terms containing derivatives $\partial_{v}$ we also use information from the grid points $\left(v_{i}, u_{i-2}\right)$ and $\left(v_{i-1}, u_{i-2}\right)$ (which is necessary to maintain second order convergence). The numerical evolution proceeds along successive lines $v=$ const, from $v_{0}$ to large values of $v$ ("null infinity"), where along each such line the integration proceeds from $u=u_{0}$ to large values of $u$ (the "event horizon").

Obviously, we do not know how to prescribe exact initial data for our problem. (In a our stationary scenario, knowledge of the exact solution at one particular moment would be equivalent to having at hand the solution at all times!) This is a standard problem of Numerical Relativity. However, unlike the situation in fully non-linear simulations of (say) equal-mass black hole mergers, here we have the luxury of being able to stably run our evolution for as long as it takes for any effect of "corrupted" initial data to dissipate off the numerical domain, and for the solution to settle down to its correct, "physical" value. To explain the basic idea, consider first the case of a scalar field, where the evolution is not constrained. Suppose that we prescribe some initial data on the initial surface (e.g., in our characteristic initial data formulation, determine the magnitude of the scalar field along $v=v_{0}$ and $\left.u=u_{0}\right)$, and then numerically integrate the inhomogeneous scalar field equation, with a source particle. Suppose that the solution we thus obtain represents, in the continuum limit, an exact solution of the inhomogeneous field equation. This solution would then differ from the true, "physical" solution by a homogeneous solution of the field equation. We may hence view the numerical solution as a superposition of the "physical" solution and a spurious homogeneous perturbation. However, homogeneous (i.e., vacuum) perturbations of black hole spacetimes always decay at late time (the "no hair" theorem). One need only wait long enough until the spurious waves radiate away and the "true" solution is revealed. On theoretical ground, we expect the spurious waves to die off in time with a power-law tail, where the power index depends on the multipole number of the mode in question. In the circular orbit case, experiment shows that one practically has to wait for about one orbital period before the spurious waves clear out (see [34,21,37], and also Figs. 3-7 below).

The above picture becomes slightly more involved in our case, where the evolving fields are components of the MP, which are subject to certain constraints in the form of gauge conditions. The initial data are now no longer freely specifiable, but are required to satisfy the gauge conditions - or otherwise our solution would not be guaranteed to satisfy the gauge conditions even at late time. Alternatively, one may incorporate in the numerical evolution procedure a machinery for damping out constraint violations, like the one described above.

Here we follow the second strategy: We use a version of the field equations that has constraint damping built into it [i.e., above Eqs. (17)], and free ourselves from the need to devise exact initial data. This option has two obvious advantages: Firstly, the required exact initial data would depend on the source in question, and will have to be developed case by case. On the other hand, once an effective constraint-damping scheme is established for circular orbits, it should be equally effective in other cases (say, eccentric orbits). Secondly, and more importantly, a successful constraint-damping scheme should take care of both initial constraint violations, and constraint violations 
from numerical errors. Specifying constraint-obeying initial data, on the other hand, would not guarantee, by itself, that constraint violations due to numerical (e.g., finite differentiation) errors remain small.

As initial data for our numerical evolution we take, most simply,

$$
\bar{h}^{(i) l m}\left(v=v_{0}\right)=\bar{h}^{(i) l m}\left(u=u_{0}\right)=0
$$

for any $l, m$ and each of $i=1, \ldots, 10$.

\section{F. Sample numerical results}

Figures 3-7 show typical numerical solutions for the various functions $\bar{h}^{(i) l m}$. For these plots we have taken the particle to move in a strong-field circular geodesic orbit at $r_{0}=7 M$ (corresponding to a period of $T_{\text {orb }} \sim 116 M$ ). The fields are evolved for an amount of time equivalent to $5 T_{\text {orb}}$, which we find more than sufficient to allow the transient spurious waves die off. The linear resolution for these runs is set at a few grid points per $M$ in both $v$ and $u$, which translates to a few hundreds points per wave cycle at $m=1$. With this resolution, the magnitude of the various $\bar{h}^{(i) l m}$ 's is resolved at a fractional accuracy better than $\sim 10^{-4}$ even near the particle's location, where the fields' gradients are largest (cf. Fig. 8 in the next section). With these specifications, a single $l, m$ mode takes of order a second to run on a standard laptop.

The graphs in Figs. 3-6 show the various $\bar{h}^{(i) l m}$ 's for $l=2$ and $m=1,2$. Plotted are the absolute values, $\left|\bar{h}^{(i) l m}\right|=\left\{\left[\operatorname{Re}\left(\bar{h}^{(i) l m}\right)\right]^{2}+\left[\operatorname{Im}\left(\bar{h}^{(i) l m}\right)\right]^{2}\right\}^{1 / 2}$. The different Figures present different slice-cuts through the 2dimensional numerical grid: $r=\operatorname{const}(=7 M), t=$ const, $u=$ const (i.e., an outgoing ray approaching null infinity), and $v=$ const (an incoming ray approaching the event horizon). Note that in Fig. 3 we show the fields $\bar{h}^{(i) l m}$ evaluated at the particle's location; recall these fields are continuous and admit definite values there. The last plot, in Fig. 7, shows the numerical solutions for $l=10$ and $m=9,10$. High- $l$ calculations are more demanding computationally, as the spacial scale of variation of the fields decreases with increasing $l$. In its present from, our code can handle multipoles up to $l \sim 20$; this should be sufficient for high precision calculations of the SF, through the mode-sum scheme.

Here are some features to notice when examining Figs. 3-7: (i) The early stage of the fields' evolution is dominated by spurious waves associated with the imperfection of the initial data. These are transients, and die off almost entirely within one orbital period of evolution time (the rate of decay of the spurious waves seems roughly independent of $l, m)$. Typically, one can safely read off the values of the fields after $\sim 2 T_{\text {orb }}$ of evolution time. In performing precision calculations (e.g., of the SF) for periodic orbits, it is easy (and advisable) to monitor the level of "contamination" from transient waves, by comparing the values of the fields at, say, $t=T_{\text {orb }}, 2 T_{\text {orb }}, 3 T_{\text {orb }}, \ldots$ (ii) As emphasized above, the fields $\bar{h}^{(i) l m}$ are all continuous through the particle's location. This is manifested in Figs. 4, 5, 6, and 7. Those fields $\bar{h}^{(i) l m}$ whose evolution equations have non-vanishing source terms (in our circular-orbit case, $i=1,3,4,6,7,8$ ) have discontinuous spacial derivatives across the particle, as expected on theoretical grounds. Those functions that are sourceless $(i=2,5,9,10)$ have continuous derivatives there. The values of the fields and their gradients at the particle provide sufficient information for calculating the gravitational SF. Our code resolves these values with great accuracy. (iii) The various $\bar{h}^{(i) l m}$ 's approach finite values at null infinity and toward the event horizon, as they are designed to do by construction. These finite values are generally non-zero, except that $\bar{h}^{(3) l m}$ vanishes toward both ends. The reason for the vanishing of $\bar{h}^{(3) l m}$ at the horizon has been explained above [see the discussion surrounding Eq. (22)]. The reason for the vanishing of this function at $r \rightarrow \infty$ will be discussed in Sec. IV C below.

Finally, we remind that, given the $\bar{h}^{(i) l m}$ 's, the MP itself is constructed algebraically through formula (19).

\section{CODE VALIDATION}

In this Section we present a series of tests we have performed to check the validity of our numerical evolution code. These include (i) a test of numerical convergence, (ii) confirmation that the numerical solutions satisfy the Lorenz gauge conditions, and (iii) comparison of the flux of energy radiated in gravitational waves to infinity, as extracted from our solutions, with the values obtained using other methods. The second and third of these tests check both our new formulation of the MP equations, and the validity of its numerical implementation. 

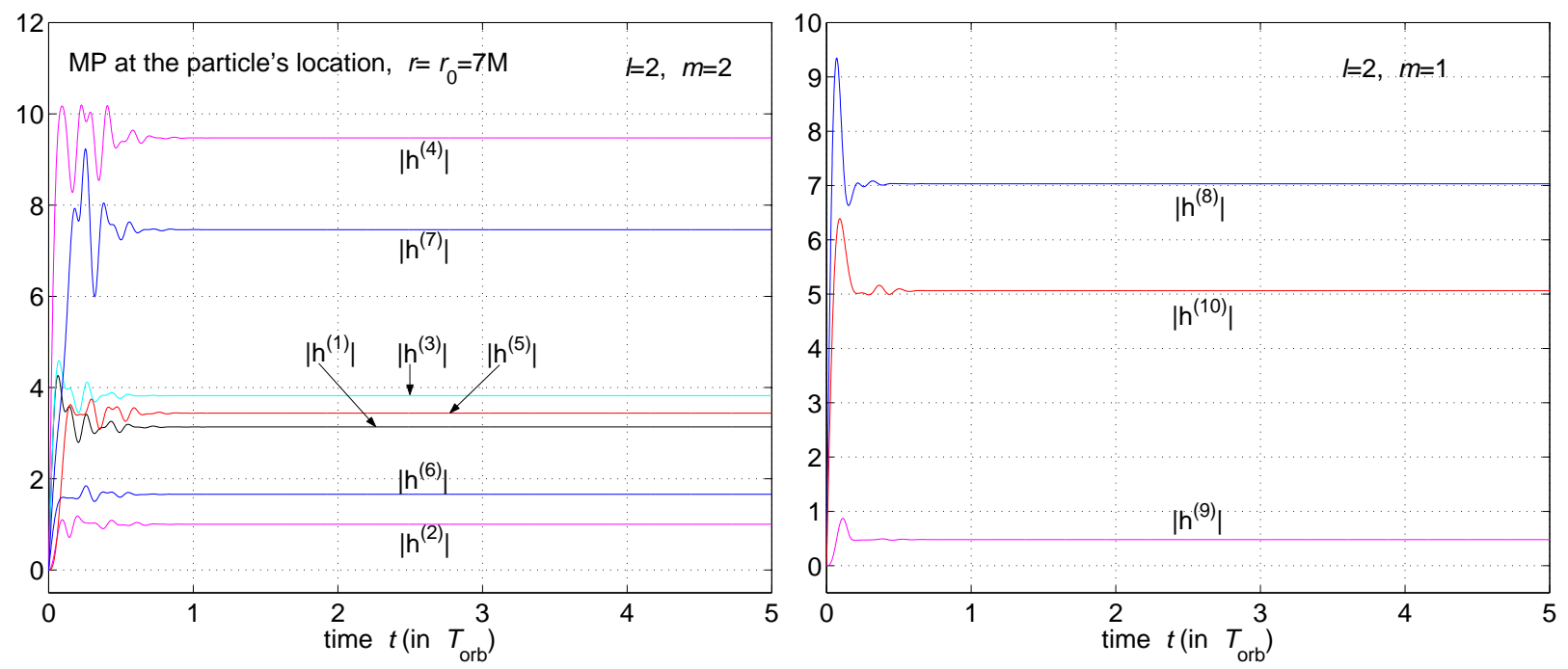

FIG. 3. Numerical solutions for the (dimensionless) Lorenz-gauge MP functions $\bar{h}^{(i) l=2, m=1,2}$, evaluated at the particle's location, for a particle in a circular orbit at $r=r_{0}=7 M$. In the Lorenz gauge (unlike in the Regge-Wheeler gauge, for example) the mode-decomposed MP is continuous at the particle, and has a definite value there. The early stage of the time evolution is dominated by transient spurious waves associated with the imperfection of the initial data. This part of the evolution (which, of course, is to be discarded in interpreting the physics content of the numerical results) lasts around one orbital period of evolution time, after which the inherent physical behavior is unveiled. Our code evolves separately the real and imaginary parts of the complex functions $\bar{h}^{(i) l m}$, which are both needed to construct the full MP [through formula (19)]; for compactness, we show here only the moduli $\left|\bar{h}^{(i) l m}\right|=\left\{\left[\operatorname{Re}\left(\bar{h}^{(i) l m}\right)\right]^{2}+\left[\operatorname{Im}\left(\bar{h}^{(i) l m}\right)\right]^{2}\right\}^{1 / 2} . \operatorname{The}$ mode $l=m=2$ is of even parity, and is constructed from the seven even-parity functions $\bar{h}^{(1, \ldots, 7)}$; the odd-parity mode $l=2, m=1$ is constructed from the three remaining functions, $\bar{h}^{(8,9,10)}$.

\section{A. Numerical convergence}

Our code is second-order convergent in the fields $\bar{h}^{(i)}$. This is demonstrated in Figs. 8 and 9 for the fields $\bar{h}^{(4)}$ and $\bar{h}^{(8)}$. (We have chosen these two functions for our demonstration here since the evolution of these specific fields couples to that of all other fields $\bar{h}^{(i)}$.)

\section{B. Preservation of the Lorenz gauge conditions}

As discussed above, we do not impose the Lorenz gauge conditions actively in the numerical evolution scheme. It is therefore important to verify that (or, rather, monitor how well) our numerical solutions indeed satisfy these four conditions, given by $H_{1}=H_{2}=H_{3}=H_{4}=0$ [see Eqs. (16)].

To check this, our code contains a subroutine that constructs the functions $H$ (out of the $\bar{h}^{(i)}$ 's and their first derivatives) along specified slice cuts through the two-dimensional numerical grid. If the gauge conditions are fully damped along the specified slice cut, we should expect all of these four quantities to converge to zero with decreasing numerical step size (and, since the $H$ 's are calculated to second-order accuracy, we expect this convergence to be quadratic). On the other hand, if there exists a finite-size constraint violation, one or more of the $H$ 's should converge (quadratically, again) to a non-zero value.

Fig. 10 shows the behavior of the four functions $H$ along an outgoing ray at late retarded time $\left(u=3 T_{\text {orb }}\right)$, in the example of the modes $l=m=2$ and $l=2, m=1$. The functions $H_{1}, H_{2}$, and $H_{3}$ are constructed from the even-parity MP modes, and are not trivially zero for $l=m=2$. The function $H_{4}$ is constructed from the odd-parity MP modes, and is not trivially zero for $l=2, m=1$. We normalize the $H$ 's (which have units of $1 /$ distance) by $(m \omega)^{-1}$, the typical length scale on which the MP varies (away from the particle). The plots demonstrate that, at least in the part of the evolution later than $t \sim T_{\text {orb}}$, all four gauge conditions are satisfied with great accuracy, to within numerical error. Similar results are obtained when examining the late time behavior along incoming rays (nearing the horizon) or along slices of fixed $r$.

We emphasize that the non-zero values of the functions $H$ in Figs. 10 are not associated with constraint violations that have not yet been fully damped - these would have tended to vanish rapidly with increasing (advanced) time. Rather, the finite-size values of the $H$ 's are merely discretization errors (coming both from the calculation of the MP fields $\bar{h}^{(i)}$, and from the construction of the $H$ 's), which tend to zero with increasing numerical resolution. Similarly, 

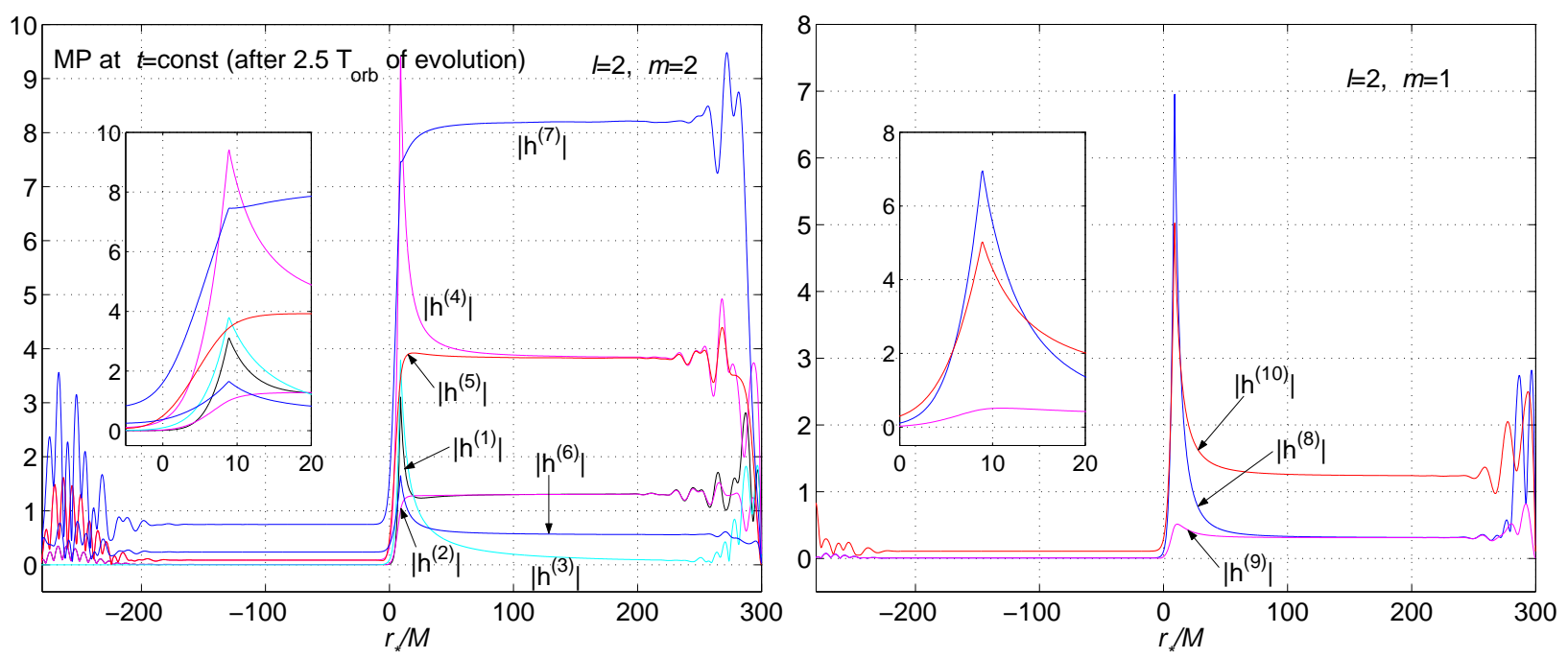

FIG. 4. A different slice cut through the solutions of Fig. 3, this time showing the behavior of the fields $\bar{h}^{(i) l=2, m=1,2}$ on a $t=$ const slice, $2.5 T_{\text {orb }}$ into the evolution. The wavy feature on the left- and right-hand sides (small and large values of $r_{*}$, respectively) are associated with the spurious initial waves propagating inward (toward the black hole) and outward (to infinity), and are to be discarded. While all functions $\bar{h}^{(i) l m}$ are continuous across the particle (located at $r=7 M, r_{*} \simeq 8.83 M$ ), those functions whose corresponding sources $S^{(i) l m}$ are non-zero have discontinues $r$ derivatives there. The fields $\bar{h}^{(2,5,9) l m}$, whose sources $S^{(2,5,9) l m}$ vanish, have continuous derivatives across the particle. The insets expand the peak area of the plots, for better clarity. The code resolves the gradients of the MP fields at the particle with good accuracy (cf. Figs. 8 in the next section). These gradients are needed for SF calculations. Note the significant damping in the MP amplitude at $r_{*} \lesssim 0$. This, presumably, is the effect of the well known potential barrier surrounding the black hole. The high-frequency spurious waves, on the other hand, penetrate this barrier with ease.

the large numerical values of the $H$ 's near the particle should not be interpreted as indicative of a higher level of constraint violation there, since at the particle, too, all functions $H$ converge to zero with increasing resolution. (Obviously, larger numerical errors arise in the $H$ 's near the particle, since the field gradients are larger there.)

\section{Energy flux at the wave zone}

Further tests of our code could be performed by comparing with other calculations of the Lorenz-gauge MP. The only such calculation we are aware of was carried out by Pfenning and Poisson [38], for a particle in a weak-field orbit. However, the analysis in [38] is mainly concerned with the SF acting on the particle, and does not provide expressions for the MP itself. Another option is to compare gauge invariant quantities derived from the MP. One such quantity is the flux of energy radiated to infinity in gravitational waves. For circular orbits in Schwarzschild, the energy flux (as distributed among the $l, m$ modes) was computed previously by Poisson [30] via frequency-domain numerical integration of the perturbation equations in the standard Regge-Wheeler gauge. More recently, Martel [37] reproduced these fluxes (as well as the fluxes from eccentric orbits) using a time-domain analysis of the perturbation equations, still in the Regge-Wheeler gauge. Here we shall compare the energy fluxes constructed from our Lorenzgauge solutions, with those obtained by Poisson and Martel.

We shall first need an expression for the energy flux at infinity in terms of the Lorenz-gauge MP. Let $\dot{E}^{\infty}$ denote the total energy per unit time crossing (outward) the 2-sphere $r=$ const where $r$ is very large. We assume that $r$ is large enough that the above 2-sphere resides in the "wave-zone", where the radius of curvature $\mathcal{R}$ is much larger than the longest wavelength $\tilde{\lambda}$ of the MP, and the perturbation takes the form of plane gravitational waves. The overdot in $\dot{E}_{\infty}$, and in the expressions below, may stand for either $\partial_{t}$ or $\partial_{u}$ (both taken with fixed $r$ ), since the two are equal at the wave zone limit. Note also that at the wave zone we can replace $h_{\alpha \beta, r}$ (fixed $t$ ) with $-h_{\alpha \beta, t}($ fixed $r$ ), and covariant derivatives with ordinary (partial) derivatives, the latter two differing by an amount of $O(\tilde{\lambda} / \mathcal{R})$.

The flux of energy in the gravitational waves can be obtained from Isaacson's effective energy-momentum tensor, $\mathcal{T}_{\alpha \beta}$, as explained, e.g., in Appendix B of Ref. [37]. However, we must use caution here: The standard expression for $\mathcal{T}_{\alpha \beta}$, as derived in [39] and used extensively in the literature (e.g., [37]), assumes that the MP is given in a gauge 



FIG. 5. Another slice cut through the solutions of Figs. 3 and 4 , this time showing the behavior along an outgoing ray, $u=\operatorname{const}\left(=3 T_{\mathrm{orb}}\right)$. Once again, the insets expand the peak areas. All fields $\bar{h}^{(i) l m}$ approach constant values at late advanced times ("null infinity"). The fluxes of energy and angular momentum carried by gravitational waves to infinity are straightforwardly extracted from these values (if fact, only $\left|\bar{h}^{(7) l m}\right|$ and $\left|\bar{h}^{(10) l m}\right|$ are needed for this purpose), as we discuss in Sec. IV C. Note how at large $v$ we seem to have $\left|\bar{h}^{(3)}\right| \sim 0,\left|\bar{h}^{(2)}\right| \sim\left|\bar{h}^{(1)}\right|,\left|\bar{h}^{(4)}\right| \sim\left|\bar{h}^{(5)}\right|$, and $\left|\bar{h}^{(8)}\right| \sim\left|\bar{h}^{(9)}\right|$. In Sec. IV C we explain how these empirical asymptotic relations are predicted theoretically.

where it is traceless. Our Lorenz gauge MP is generally not traceless ${ }^{8}$ and we will need to generalize the expression for the effective energy-momentum tensor to the case $h \neq 0$. This can be done by repeating Isaacson's derivation, starting at his Eq. (2.4) and going through the averaging procedure described in his analysis - but this time keeping track of all the trace terms. The result is

$$
\mathcal{T}_{\mu \nu}=\frac{1}{32 \pi}\left\langle\bar{h}_{; \mu}^{\alpha \beta} \bar{h}_{\alpha \beta ; \nu}-\frac{1}{2} \bar{h}_{, \mu} \bar{h}_{, \nu}\right\rangle
$$

where $\langle\cdots\rangle$ denotes an average over a region of spacetime much larger than $\tilde{\lambda}$. The first term in this expression (which is invariant under $\left.\bar{h}_{\alpha \beta} \rightarrow h_{\alpha \beta}\right)$ is the standard Isaacson tensor. The second term is a necessary correction when the MP has a non-zero trace. The form (53) is now truly gauge invariant (unlike Isaacson's original formula) and can be used to extract the energy flux from the Lorenz-gauge MP. Given the effective energy-momentum tensor, the energy flux at infinity is given by [37]

$$
\dot{E}_{\infty}=-r^{2} \int d \Omega \mathcal{T}_{t r}
$$

where the integration is carried over the above mentioned 2-sphere. Note that the integration over $d \Omega$ automatically takes care of averaging $\mathcal{T}_{\mu \nu}$ over a scale $\sim \mathcal{R}$, so one can effectively ignore the averaging procedure in Eq. (53) when evaluating the flux.

We next consider the distribution of $\dot{E}_{\infty}$ among the different multipoles. To this end, we substitute for the MP in Eq. (53) using the expansion (8). In each of the two quadratic terms we formally replace one of the MP factors by its complex conjugate (which is allowed since the MP is a real function). One can sort the resulting terms inside the integral $\int d \Omega$ into 3 groups, according to their angular dependence: One group of terms comes with $Y^{l^{\prime} m^{\prime}} Y^{l m *}$, the other with $Y_{, \theta}^{l^{\prime} m^{\prime}} Y_{, \theta}^{l m *}+\sin ^{-2} \theta Y_{, \varphi}^{l^{\prime} m^{\prime}} Y_{, \varphi}^{l m *}$, and the third with $\left(D_{2} Y^{l^{\prime} m^{\prime}}\right)\left(D_{2} Y^{l m *}\right)+\sin ^{-2} \theta\left(D_{1} Y^{l^{\prime} m^{\prime}}\right)\left(D_{1} Y^{l m *}\right)$. Using the orthogonality relations (A4), and replacing all derivatives $\partial_{r}$ by $-\partial_{t}$, one obtains $\dot{E}_{\infty}=\sum_{l m} \dot{E}_{\infty}^{l m}$, with the individual multipolar contributions given by

\footnotetext{
${ }^{8}$ Generally, the supplementary gauge condition $h=0$ cannot be imposed in addition to the Lorenz gauge condition (3), unless spacetime is globally vacuum - which is not our case here. That our solutions indeed have $h \neq 0$ is demonstrated in Figs. $3-7$, recalling the trace is constructed from the $\bar{h}^{(i)}$ 's through Eq. (22).
} 

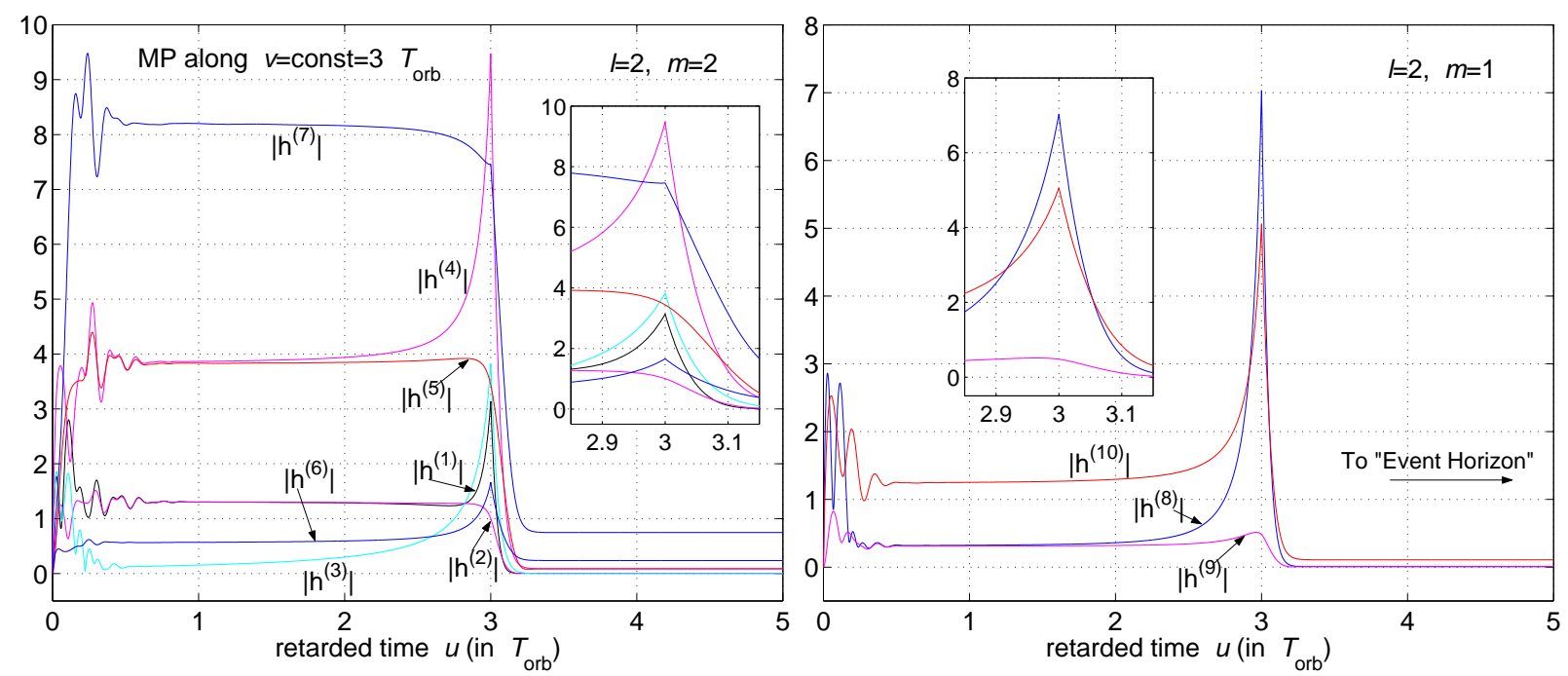

FIG. 6. One more slice cut through the solutions of Figs. 3, 4 and 5 , showing the behavior along an incoming ray, $v=$ const $\left(=3 T_{\text {orb }}\right)$. With increasing retarded time, approaching the "event horizon", all fields $\left|\bar{h}^{(i) l m}\right|$ settle at constant values. Although hard to tell from these plots, these values are generally nonzero (with the exception of $\bar{h}^{(3) l m}$, which tends to zero at the horizon-see the text for explanation).

$$
\begin{aligned}
\dot{E}_{\infty}^{l m}= & \frac{\mu^{2}}{32 \pi}\left[\frac{1}{2}\left(\left|\bar{h}_{\infty}^{(1)}\right|^{2}-\left|\bar{h}_{\infty}^{(2)}\right|^{2}+2\left|\bar{h}_{\infty}^{(3)}\right|^{2}\right)\right. \\
& -\frac{1}{4 l(l+1)}\left(\left|\bar{h}_{\infty}^{(4)}\right|^{2}-\left|\bar{h}_{\infty}^{(5)}\right|^{2}+\left|\bar{h}_{\infty}^{(8)}\right|^{2}-\left|\bar{h}_{\infty}^{(9)}\right|^{2}\right) \\
& \left.+\frac{1}{2 \lambda l(l+1)}\left(\left|\bar{h}_{\infty}^{(\overline{7})}\right|^{2}+\left|\bar{h}_{\infty}^{(\dot{1} 0)}\right|^{2}\right)\right] .
\end{aligned}
$$

Here, the subscript $\infty$ under the $\bar{h}^{(i)}$ 's indicates that these functions are to be evaluated at the wave zone.

The above expression for $\dot{E}_{\infty}^{l m}$ simplifies by virtue of the gauge conditions. Consider, for example, the condition $H_{1}=0$ [Eq. (16a)]: Neglecting non-derivative terms [which at the wave zone are $O(\tilde{\lambda} / \mathcal{R})$ times smaller than the derivative terms], and replacing $\partial_{r} \rightarrow-\partial_{t}$, we find $\bar{h}_{\infty, t}^{(2)}=-\bar{h}_{\infty, t}^{(1)}-\bar{h}_{\infty, t}^{(3)}$. Similarly, from $H_{2}=0, H_{3}=0$, and $H_{4}=0$ we find, respectively, $\bar{h}_{\infty, t}^{(2)}=-\bar{h}_{\infty, t}^{(1)}+\bar{h}_{\infty, t}^{(3)}, \bar{h}_{\infty, t}^{(4)}=-\bar{h}_{\infty, t}^{(5)}$, and $\bar{h}_{\infty, t}^{(8)}=-\bar{h}_{\infty, t}^{(9)}$. Altogether, we thus have

$$
\left|\bar{h}_{\infty}^{(2)}\right|^{2}=\left|\bar{h}_{\infty}^{(1)}\right|^{2}, \quad\left|\bar{h}_{\infty}^{(3)}\right|^{2}=0, \quad\left|\bar{h}_{\infty}^{(4)}\right|^{2}=\left|\bar{h}_{\infty}^{(5)}\right|^{2}, \quad\left|\bar{h}_{\infty}^{(8)}\right|^{2}=\left|\bar{h}_{\infty}^{(9)}\right|^{2}
$$

(In our stationary scenario we have $\left|\bar{h}_{\infty}^{\dot{(2)}}\right|^{2}=m^{2} \omega^{2}\left|\bar{h}_{\infty}^{(2)}\right|^{2}$, which means that the asymptotic relations (56) hold between the functions $\bar{h}^{(i)}$ themselves, not only between their time derivatives. That our numerical solutions satisfy these relations at large $r$ is easily visible in Figs. 4 and 5. This provides yet another validity test for our code.) Hence, Eq. (55) reduces to the final form

$$
\dot{E}_{\infty}^{l m}=\frac{\mu^{2}}{64 \pi \lambda l(l+1)}\left(\left|\bar{h}_{\infty}^{(7)}\right|^{2}+\left|\bar{h}_{\infty}^{(i 0)}\right|^{2}\right) .
$$

Note that the flux carried by each individual mode is extracted from a single $\bar{h}^{(i)}$ function: $\bar{h}^{(7)}$ in the case of even-parity modes (even $l+m$ ), or $\bar{h}^{(10)}$ in the case of odd-parity modes (odd $l+m$ ).

Table II lists the values of $\dot{E}_{l m}^{\infty}$ for $l=1, \ldots, 5$, as computed from our numerical Lorenz-gauge solutions based on Eq. (57). For this table we set the particle to move on a circular geodesic at $r_{0}=7.9456 M$, to allow comparison with Poisson and Martel, who provide results for this radius. The functions $\bar{h}^{(7)}$ and $\bar{h}^{(10)}$ are evaluated at $v=40 T_{\text {orb }}$ and $u=3 T_{\text {orb }}$, corresponding to $r \sim 2100 M$ and $t \sim 2500 M$. It is possible to estimate the error we make in extracting the flux at a finite $r$ by looking at the residual values of, e.g., $\left|\bar{h}^{(3)}\right|$ and $\left|\bar{h}^{(8)}\right|-\left|\bar{h}^{(9)}\right|$, which should vanish at the limit $r \rightarrow \infty$. If we assume that the finite-r error in $\bar{h}^{(7)}$ is the same as the largest among the residues in $\left|\bar{h}^{(3)}\right|$, $\left|\bar{h}^{(1)}\right|-\left|\bar{h}^{(2)}\right|$, and $\left|\bar{h}^{(4)}\right|-\left|\bar{h}^{(5)}\right|$, we obtain from our code that the fractional error in the flux, for even-parity modes, is typically of order $0.1-0.2 \%$. In the same way, assuming that the finite- $r$ error in $\bar{h}^{(10)}$ is the same as the residue in $\left|\bar{h}^{(8)}\right|-\left|\bar{h}^{(9)}\right|$, we expect a fractional error of order $0.01-0.3 \%$ for the various odd-parity modes. 

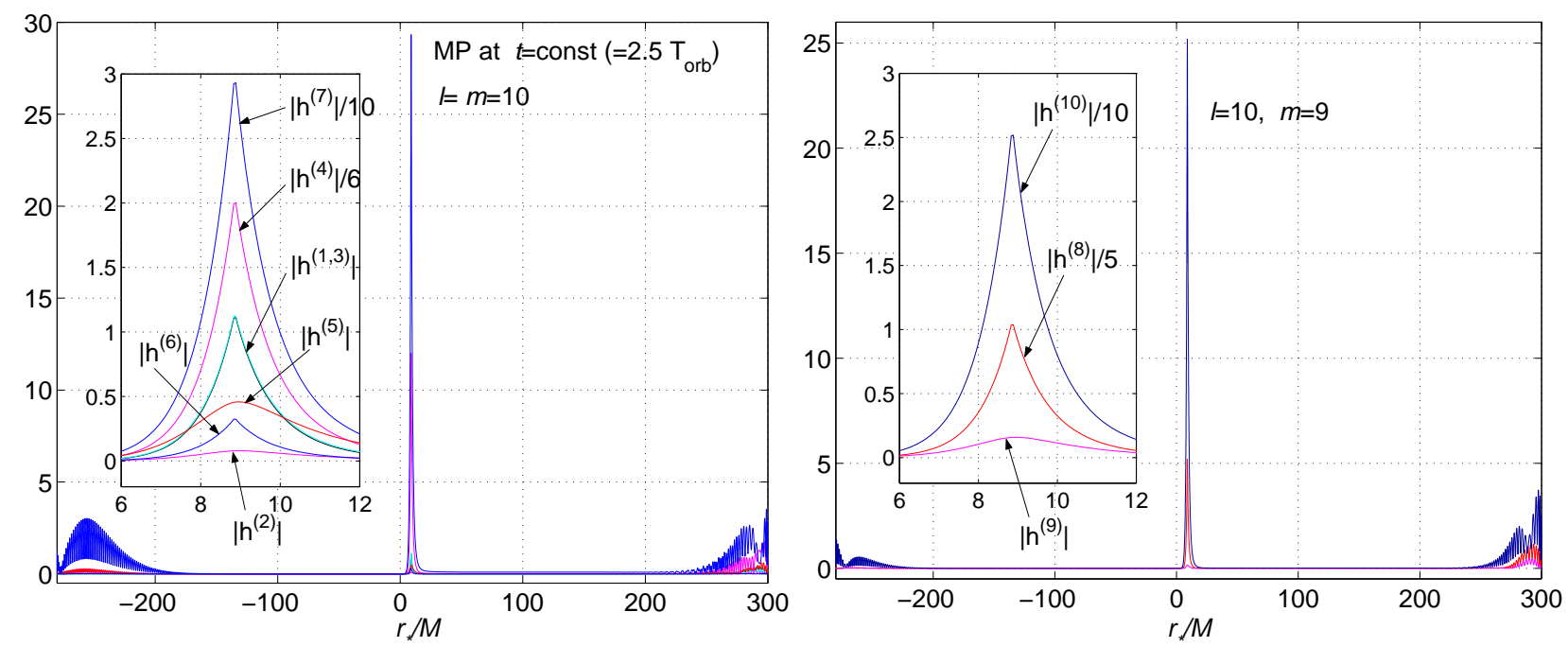

FIG. 7. The MP functions $\bar{h}^{(i) l m}$ for $l=10$ and $m=9,10$, on a $t=$ const slice (compare with Fig. 4 ). Spacial gradients of the MP get larger with growing $l$, but at $l=10$ the particle is still perfectly resolvable (our code performs well for multipole numbers up to $\sim 20$ ). The seemingly noisy features on both ends of the plots are, in fact, numerically robust: They are transient spurious waves of the type also apparent in Figs. 3-6, but now occurring at much higher frequencies.

Our code reproduces the energy fluxes of Poisson [30] and Martel [37] with very good accuracy. Our values are no more than $\sim 1 \%$ off those of Martel, and no more than $0.07 \%$ off those of Poisson. The overall energy flux (summed over the first 5 modes) agrees with that computed by Poisson with a mere $\sim 5 \cdot 10^{-5}$ relative difference.

\section{SUMMARY AND DISCUSSION OF FUTURE APPLICATIONS}

We advocate here a new approach to the computation of the metric perturbation from a small particle orbiting a black hole. The approach incorporates the following "principles": (a) The particle is represented as a delta function source term (rather than an extended distribution) in the field equations; (b) The perturbation equations are formulated and integrated for the MP itself (rather than generating functions thereof); (c) The MP is solved for in the Lorenz gauge; (d) The numerical integration of the perturbation equations is carried out in the time domain.

The main advantages offered by this approach are: (i) In applications requiring knowledge of the MP itself, one avoids the difficult issue of MP reconstruction; (ii) The Lorenz-gauge MP provides the most natural description of the field near the particle, and is the most convenient to work with in analyzing the singular structure of this field; (iii) The Lorenz-gauge MP can be incorporated immediately into existing schemes for calculating the gravitational SF. (iv) Time-domain numerical integration is more efficient in dealing with eccentric orbits than traditional frequency-domain methods, and is more easily generalizable from one set of orbits to another.

Here we developed the above approach, and explored its feasibility, for orbits in Schwarzschild. The core of our formulation includes the decoupled field equations, Eqs. (17), along with the supplementary gauge conditions, Eqs. (16). We implemented this formulation numerically for the case of circular orbits, using a characteristic time-evolution code that incorporates a constraint damping scheme. We demonstrated that such a code can efficiently resolve the singular field near the particle, in a computationally inexpensive manner.

Since our code integrates the perturbation equations in the time domain, it is possible to use it for analyzing any orbit in Schwarzschild: For any given trajectory $x_{\mathrm{p}}(\tau)$ (which, if geodesic, can always be taken to be equatorial) one merely has to specify appropriate source functions $S^{(i) l m}\left[r_{\mathrm{p}}(\tau)\right]$ in Eq. (17), and feed these functions into the subroutine in our code that tells the numerical integrator where the particle is at each step of the evolution. The numerical properties of the evolution code (stability, convergence rate, constraint damping) are insensitive to the choice of source. Since our code performs well with circular orbits, it will perform well with any other orbit.

Much more challenging is the extension to the Kerr case, which, however, also provides the main motivation for the above approach. On a Kerr background, the perturbation equations are no longer separable into multipole modes in the time domain. A time-domain integrator will therefore have to evolve the equations in $2+1$ dimensions (two spacial dimensions, and time; the azimuthal direction remains separable even in the time domain). There exist a few working codes for integrating Teukolsky's equation in the time domain, for vacuum perturbations in Kerr [see, e.g., $[40,41])]$. A similar code will have to be developed for integrating the Lorenz-gauge field equations (4), with a particle 

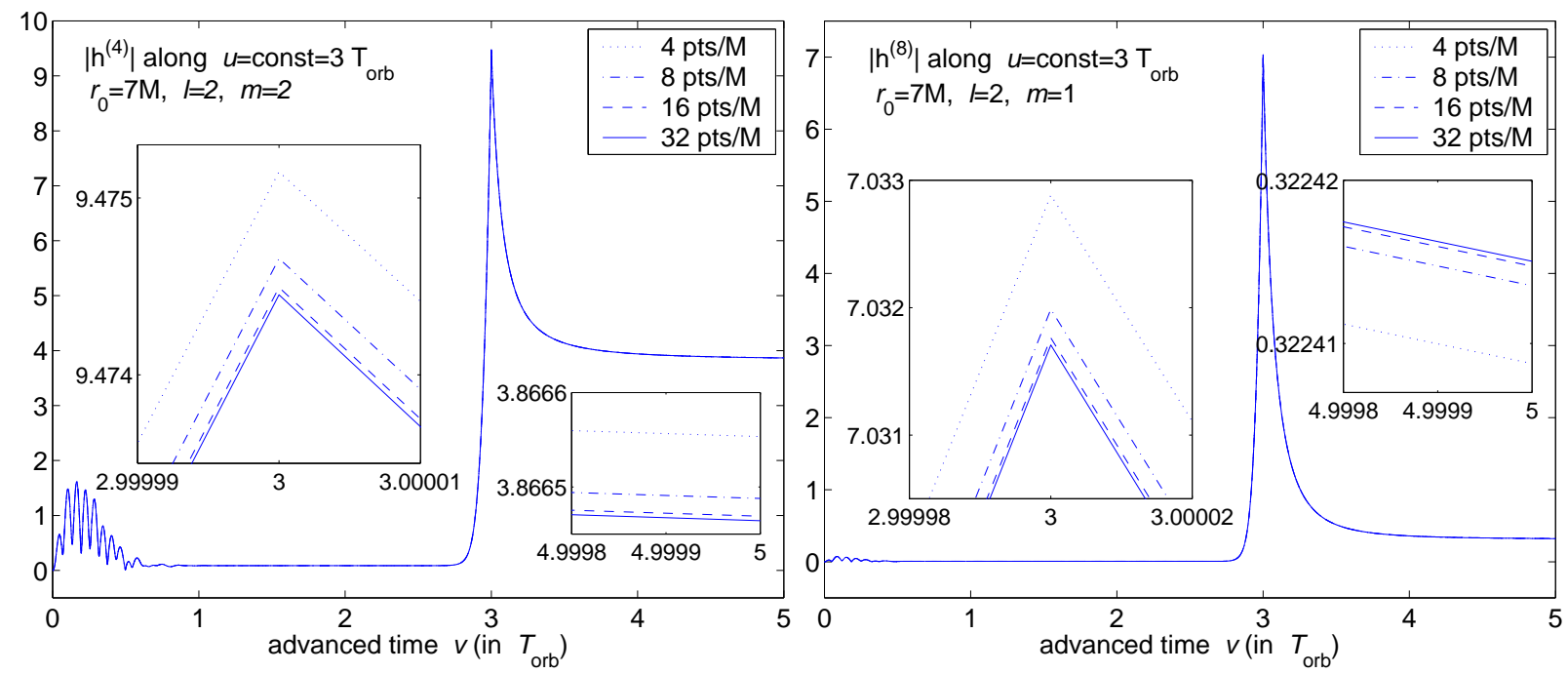

FIG. 8. Illustration of numerical convergence: Shown here are numerical solutions for (the absolute values of) the functions $\bar{h}^{(4) l=2, m=2}$ (left panel) and $\bar{h}^{(8) l=2, m=1}$ (right panel) along an outgoing ray $u=$ const $=3 T_{\text {orb }}$ - the solutions also shown in Fig. 5 . In each of the figures we have superposed 4 numerical solutions, obtained with different numerical resolutions: 4, 8, 16, and 32 grid points per $M$ (this corresponds to the linear resolution; the numbers of grid points per unit grid area $M^{2}$ are the squares of these values). The insets show, greatly magnified, two details from each plot: one near the peak (the particle's location), and the other at the far right end ("null infinity"). It clearly appears that the solutions are numerically convergent, and that the convergence rate is faster than linear. Note also that, with the range of resolutions used here, the magnitude of the fields is already resolvable to within fractional errors of $\sim 10^{-5}$. This is the case even near the particle, where the fields' gradients are largest.

source. The challenge here is not in the expected high computational expense insomuch as it is in the difficulty of treating the particle singularity: In $2+1$ dimensions the Lorenz gauge perturbation field is no longer continuous and finite near the particle (as it is, conveniently, in 1+1 dimensions). Rather, it diverges toward the particle. Although this divergence is slow (logarithmic in proper distance from the particle), it poses a serious problem when it comes to numerical implementation.

One possible way around this problem is to implement a procedure reminiscent of the "puncture" scheme often in use in Numerical Relativity. To sketch the basic idea, let us represent the field equation (4), symbolically, by ' $\square h=\delta$ ', where ' $\delta$ ' represents a given fixed trajectory on the black hole background, and the left-hand side is understood to contain also the Riemann term. We introduce a worldtube around the particle, of some fixed radius $\rho$ that we keep as a control parameter. $\rho$ should be much smaller than the background's radius of curvature, and much larger than the radius of curvature associated with the particle; say, $\rho \sim \sqrt{M \mu}$. The numerical time-evolution integrator than utilizes the following algorithm: At any point (in the 2+1-dimensional numerical grid space) outside the above worldtube, the integrator evolves the homogeneous equation $\square h=0$ as it is. However, at points inside the tube, the integrator solves for a different, "punctured" field $h_{\text {punct }} \equiv h-h_{\text {sing }}$, where $h_{\text {sing }}$ is an analytic approximation for the field $h$ near the singularity, chosen such that $h_{\text {punct }}$ is continuous everywhere inside the worldtube, including on the worldline itself. Thus, inside the worldtube one solves the equation $\square h_{\text {punct }}=\delta-\square h_{\text {sing }}$, where the source term on the right-hand side is now distributed, but 'more regular' than the original source. The evolution across the boundary of the worldtube proceeds by matching the external solution $h$ to $h_{\text {punct }}+h_{\text {sing. }}$. That this (simple) idea could work in practice has been demonstrated recently with a toy model of a scalar field in Schwarzschild [42].

As explained in the introduction, our main drive in developing the computational approach of this paper is the problem of calculating the SF. With the new computational tools at hand, freeing us from gauge complexities and issues of MP reconstruction, we can straightforwardly implement the mode-sum scheme [7]. The scheme, we remind, requires the values of each multipole of the Lorenz-gauge MP and its derivatives at the particle. The modes are then "regularized" individually, by subtracting a certain quantity, given analytically (the "regularization parameters" [7]), from each. The sum over regularized modes then gives the physical SF experienced by the particle. Our current code can be used to calculate the SF in this manner, for any orbit in Schwarzschild. One minor technical matter still to be addressed is the fact that the regularization parameters prescribed in the literature correspond (somewhat awkwardly) to a scalar-harmonic decomposition of the force's components, whereas the entities we calculate numerically are tensor harmonic components of the MP, yielding the vector-harmonic components of the force. It will be required to obtain the appropriate regularization parameters for the vector-harmonic components, or, alternatively, expand the vector harmonic components in scalar harmonics in order to allow them to communicate with the standard parameters. 

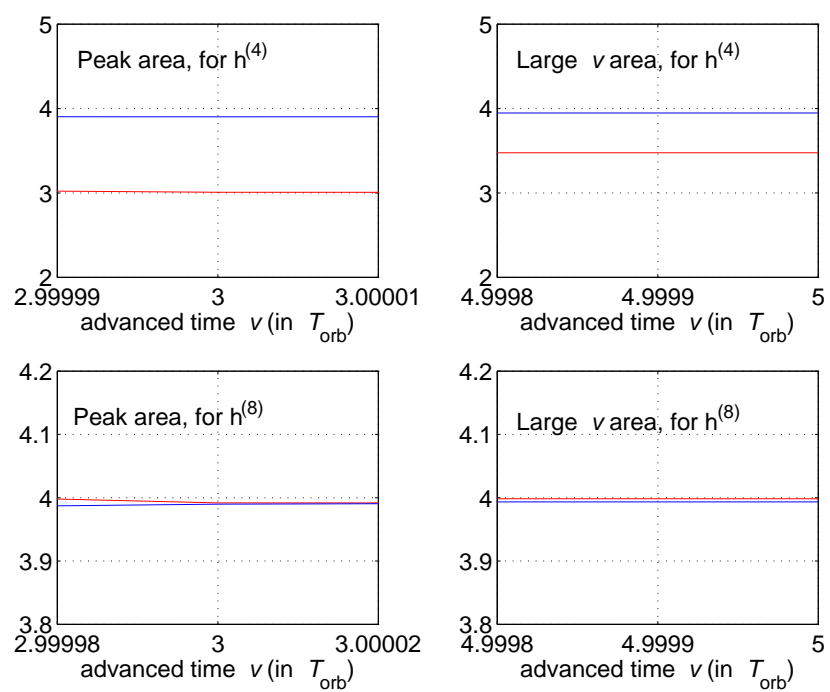

FIG. 9. Demonstration of second-order numerical convergence. These four plots provide a quantitative test of the solution's numerical convergence rate. The left and right panels of the upper (lower) row correspond, respectively, to the left and right insets in the left (right) panels of Fig. 8. The red (pale) line in each of the plots shows the ratio $\left(\left|\bar{h}^{(i)}[4 \mathrm{pts} / M]-\bar{h}^{(i)}[8 \mathrm{pts} / M]\right|\right) /\left(\left|\bar{h}^{(i)}[8 \mathrm{pts} / M]-\bar{h}^{(i)}[16 \mathrm{pts} / M]\right|\right)$, where $i=4,8$ and $\bar{h}^{(i)}[4 \mathrm{pts} / M]$, for example, represents the numerical solution obtained with resolution of 4 grid points per $M$. The blue (dark) lines similarly show the ratio $\left(\left|\bar{h}^{(i)}[8 \mathrm{pts} / M]-\bar{h}^{(i)}[16 \mathrm{pts} / M]\right|\right) /\left(\left|\bar{h}^{(i)}[16 \mathrm{pts} / M]-\bar{h}^{(i)}[32 \mathrm{pts} / M]\right|\right)$. Both ratios approach the value of 4 with growing resolution, which indicates second-order convergence.

Either of these options is essentially straightforward to implement.

\section{ACKNOWLEDGMENTS}

During the work on this project we have benefited from discussions with many, including Nils Andersson, Curt Cutler, Steve Detweiler, Jonathan Gair, Kostas Glampedakis, Yasushi Mino, and James Vickers. We are grateful to all of them. We especially thank Eric Poisson for many valuable comments, and Carsten Gundlach for useful advice on the issue of constraint damping. We gratefully acknowledge the support of the NASA Center for Gravitational Wave Astronomy at The University of Texas at Brownsville (NAG5-13396), and the NSF for financial support from grants PHY-0140326 and PHY-0354867. L.B. also acknowledges financial support from the Nuffield Foundation, and thanks the Aspen Center for Physics for hospitality during the Aspen 2005 Summer Workshop on LISA, where part of this research was carried out. 

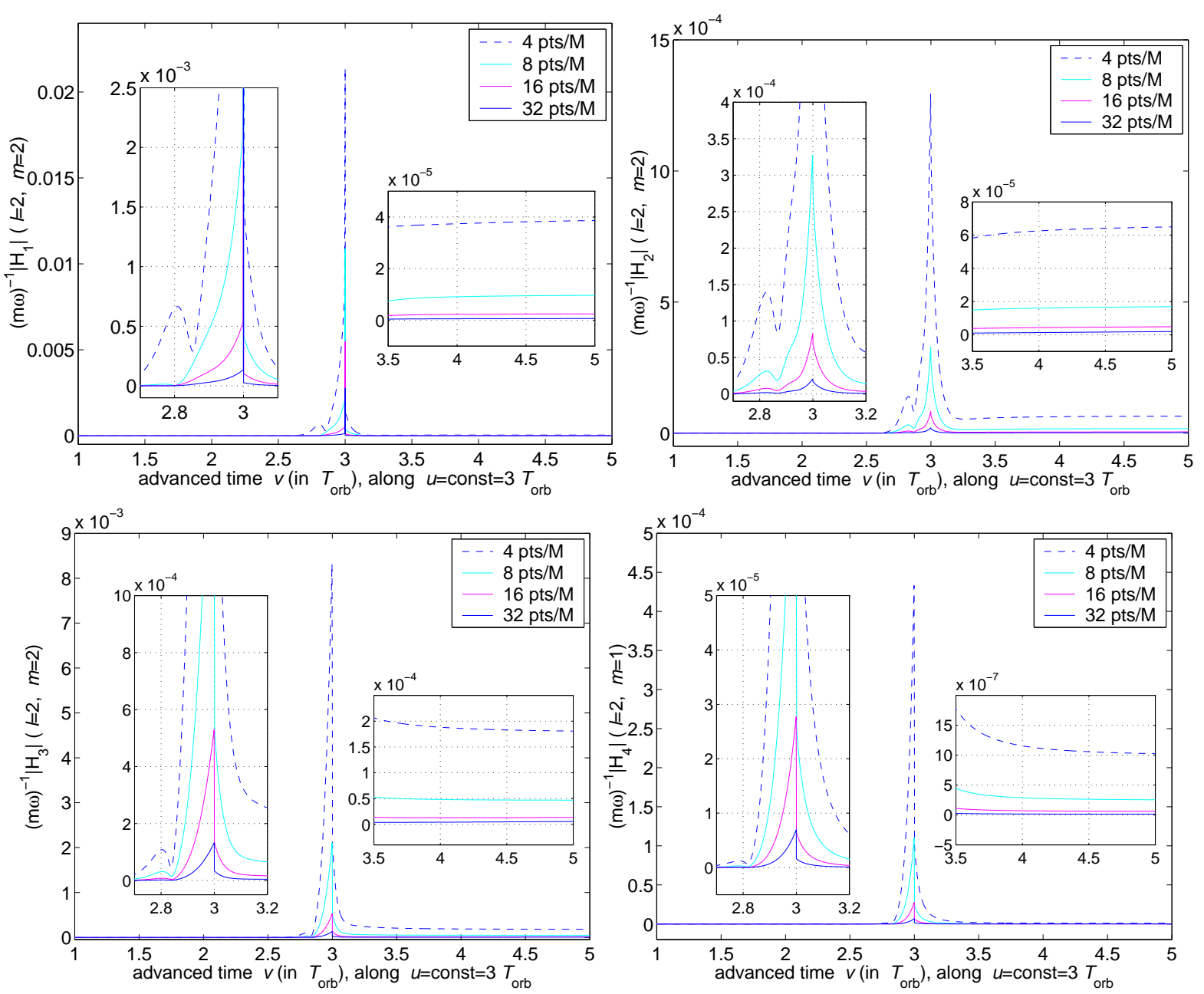

FIG. 10. Verifying that our numerical solutions satisfy the Lorenz gauge conditions. Plotted here are the (absolute values of the) four

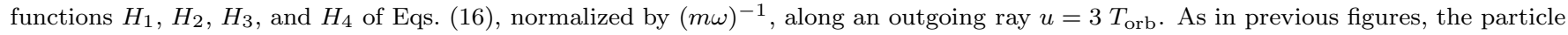
is moving on a circular geodesic orbit with $r_{0}=7 \mathrm{M}$. We consider here the modes $l=m=2$ (for $H_{1}, H_{2}$, and $H_{3}$, which involve the even-parity MP modes) and $l=2, m=1$ (for $H_{4}$, which involves the odd-parity MP modes). The Lorenz gauge condition is satisfied if (and only if) all four functions $H$ vanish at the limit of vanishing step size. In each of the plots we show, superposed, a sequence of four lines, obtained with decreasing step sizes. The insets magnify specific areas in each of the plots. All four functions $H$ appear to converge to values very close to zero, indicating that the gauge conditions are satisfied with good accuracy. See the text for further discussion.

\section{APPENDIX A: BASIS OF TENSOR HARMONICS}

The tensor harmonics $Y_{\alpha \beta}^{(i) l m}$ adopted in this paper are given, in Schwarzschild coordinates $t, r, \theta, \varphi$, by

$$
\begin{gathered}
Y_{\alpha \beta}^{(1)}=\frac{1}{\sqrt{2}}\left(\begin{array}{cccc}
1 & 0 & 0 & 0 \\
0 & f^{-2} & 0 & 0 \\
0 & 0 & 0 & 0 \\
0 & 0 & 0 & 0
\end{array}\right) Y^{l m}, \quad Y_{\alpha \beta}^{(2)}=\frac{f^{-1}}{\sqrt{2}}\left(\begin{array}{cccc}
0 & 1 & 0 & 0 \\
1 & 0 & 0 & 0 \\
0 & 0 & 0 & 0 \\
0 & 0 & 0 & 0
\end{array}\right) Y^{l m}, \quad Y_{\alpha \beta}^{(3)}=\frac{1}{\sqrt{2}}\left(\begin{array}{cccc}
1 & 0 & 0 & 0 \\
0 & -f^{-2} & 0 & 0 \\
0 & 0 & 0 & 0 \\
0 & 0 & 0
\end{array}\right) Y^{l m}, \\
Y_{\alpha \beta}^{(4)}=\frac{r}{\sqrt{2 l(l+1)}}\left(\begin{array}{cccc}
0 & 0 & \partial_{\theta} & \partial_{\varphi} \\
0 & 0 & 0 & 0 \\
\partial_{\theta} & 0 & 0 & 0 \\
\partial_{\varphi} & 0 & 0 & 0
\end{array}\right) Y^{l m}, \quad Y_{\alpha \beta}^{(5)}=\frac{r f^{-1}}{\sqrt{2 l(l+1)}}\left(\begin{array}{cccc}
0 & 0 & 0 & 0 \\
0 & 0 & \partial_{\theta} & \partial_{\varphi} \\
0 & \partial_{\theta} & 0 & 0 \\
0 & \partial_{\varphi} & 0 & 0
\end{array}\right) Y^{l m}
\end{gathered}
$$




\begin{tabular}{|c|c|c|c|c|c|c|}
\hline$l$ & $m$ & $\begin{array}{c}\dot{E}_{l m}^{\infty}, \text { this paper: } \\
t \text {-domain, from } h_{\alpha \beta}^{\text {Lorenz }} \\
\end{array}$ & $\begin{array}{r}\dot{E}_{l m}^{\infty}, \text { Pois } \\
f \text {-domain, }\end{array}$ & $\begin{array}{l}\mathrm{n}[30]: \\
\text { om } h_{\alpha \beta}^{\mathrm{RW}} \\
\end{array}$ & $\begin{array}{r}\dot{E}_{l m}^{\infty}, \mathrm{Ma} \\
t \text {-domain, }\end{array}$ & $\begin{array}{l}\text { el [37]: } \\
\text { om } h_{\alpha \beta}^{\mathrm{RW}}\end{array}$ \\
\hline 2 & 1 & $8.1654 e-07$ & $8.1633 e-07$ & $|-0.03 \%|$ & $8.1623 e-07$ & $-0.04 \%$ \\
\hline & 2 & $1.7061 e-04$ & $1.7063 e-04$ & [+0.01\%] & $1.7051 e-04$ & {$[-0.06 \%]$} \\
\hline 3 & 1 & $2.1734 e-09$ & $2.1731 e-09$ & {$[-0.01 \%]$} & $2.1741 e-09$ & {$[+0.03 \%]$} \\
\hline & 2 & $2.5207 e-07$ & $2.5199 e-07$ & {$[-0.03 \%]$} & $2.5164 e-07$ & {$[-0.17 \%]$} \\
\hline & 3 & & $2.5471 e-05$ & {$[-0.03 \%]$} & $2.5432 e-05$ & {$[-0.18 \%]$} \\
\hline 4 & 1 & 8.3 & 8.39 & {$[-0.1$} & $8.3507 e-13$ & {$[-0.57 \%]$} \\
\hline & 2 & 2.5 & 2.50 & {$[-0$.} & 2.498 & {$[-0.45 \%]$} \\
\hline & 3 & 8 & 5.77 & {$[-0.1$} & $5.7464 e-08$ & {$[-0.51 \%]$} \\
\hline & 4 & $4.7284 e-06$ & $4.7256 e-06$ & {$[-0.06 \%]$} & $4.7080 e-06$ & {$[-0.43 \%]$} \\
\hline 5 & 1 & $1.2598 e-15$ & $1.2594 e-15$ & {$[-0.03 \%]$} & $1.2544 e-15$ & {$[-0.43 \%]$} \\
\hline & 2 & $2.7877 e-12$ & $2.7896 e-12$ & [+0.07\%] & $2.7587 e-12$ & {$[-1.04 \%]$} \\
\hline & 3 & 09 & 1.0933 & {$[-0.01 \%]$} & $1.0830 e-09$ & {$[-0.95 \%]$} \\
\hline & 4 & & 1.2324 & {$[+0 .(1$} & $1.2193 e-08$ & {$[-1.02 \%]$} \\
\hline & 5 & 9.46 & $9.4563 e-07$ & {$[-0.06 \%]$} & $9.3835 e-07$ & {$[-0.83 \%]$} \\
\hline & & $2.0291-04$ & $2.0292 e-04$ & +0.00 & $2.0273 e-04$ & -0.09 \\
\hline
\end{tabular}

TABLE II. Comparison of energy fluxes per $l, m$-mode at infinity, as extracted from our Lorenz-gauge MP, with the values obtained using other methods. The values in the table give $\dot{E}_{l m}^{\infty}$ [in units of $(\mu / M)^{2}$ ] for $r_{0}=7.9456$, with $m<0$ modes folded over onto $m>0$. The results from Poisson's and Martel's computations are quoted from Ref. [37]. Both Poisson and Martel extracted their fluxes from the MP in the Regge-Wheeler gauge, the former using frequency domain analysis and the latter integrating the field equations in the time domain. The values under "this paper" are derived using Eq. (57), with the Lorenz-gauge functions $\bar{h}^{(7)}$ (for modes with even $\left.l+m\right)$ and $\bar{h}^{(10)}$ (for modes with odd $l+m$ ) extracted at $v=40 T_{\text {orb }}$ and $u=3 T_{\text {orb }}$ (corresponding to $r \sim 2100 M$ and $t \sim 2500 M$ ). The values in square brackets under "Poisson" and "Martel" give the relative difference, in percents, between their results and ours.

$$
\begin{aligned}
& Y_{\alpha \beta}^{(6) l m}=\frac{r^{2}}{\sqrt{2}}\left(\begin{array}{cccc}
0 & 0 & 0 & 0 \\
0 & 0 & 0 & 0 \\
0 & 0 & 1 & 0 \\
0 & 0 & 0 & s^{2}
\end{array}\right) Y^{l m}, \quad Y_{\alpha \beta}^{(7) l m}=\frac{r^{2}}{\sqrt{2 \lambda l(l+1)}}\left(\begin{array}{cccc}
0 & 0 & 0 & 0 \\
0 & 0 & 0 & 0 \\
0 & 0 & D_{2} & D_{1} \\
0 & 0 & D_{1} & -s^{2} D_{2}
\end{array}\right) Y^{l m}, \\
& Y_{\alpha \beta}^{(8) l m}=\frac{r}{\sqrt{2 l(l+1)}}\left(\begin{array}{cccc}
0 & 0 & s^{-1} \partial_{\varphi} & -s \partial_{\theta} \\
0 & 0 & 0 & \\
s^{-1} \partial_{\varphi} & 0 & 0 & 0 \\
-s \partial_{\theta} & 0 & 0 & 0
\end{array}\right) Y^{l m} \\
& Y_{\alpha \beta}^{(9) l m}=\frac{r f^{-1}}{\sqrt{2 l(l+1)}}\left(\begin{array}{cccc}
0 & 0 & 0 & 0 \\
0 & 0 & s^{-1} \partial_{\varphi} & -s \partial_{\theta} \\
0 & s^{-1} \partial_{\varphi} & 0 & 0 \\
0 & -s \partial_{\theta} & 0 & 0
\end{array}\right) Y^{l m} \\
& Y_{\alpha \beta}^{(10) l m}=\frac{r^{2}}{\sqrt{2 \lambda l(l+1)}}\left(\begin{array}{cccc}
0 & 0 & 0 & 0 \\
0 & 0 & 0 & 0 \\
0 & 0 & s^{-1} D_{1} & -s D_{2} \\
0 & 0 & -s D_{2} & -s D_{1}
\end{array}\right) Y^{l m}
\end{aligned}
$$

where $f \equiv(1-2 M / r), Y^{l m}(\theta, \varphi)$ are the standard scalar spherical harmonics, $s \equiv \sin \theta, \lambda \equiv(l-1)(l+2)$, and the angular operators $D_{1}$ and $D_{2}$ are given by

$$
D_{1} \equiv 2\left(\partial_{\theta}-\cot \theta\right) \partial_{\varphi}, \quad D_{2} \equiv \partial_{\theta \theta}-\cot \theta \partial_{\theta}-s^{-2} \partial_{\varphi \varphi}
$$

The radial factors involving $r$ and $f$ are introduced for dimensional balance and for settling the horizon behavior. The harmonics $Y_{\alpha \beta}^{(i) l m}$ constitute an orthonormal set, in the sense expressed in Eq. (7). This can be readily verified based on the identities

$$
\int Y^{l^{\prime} m^{\prime}} Y^{l m *} d \Omega=\delta_{l l^{\prime}} \delta_{m m^{\prime}}
$$




$$
\begin{gathered}
\int\left(Y_{, \theta}^{l^{\prime} m^{\prime}} Y_{, \theta}^{l m *}+\sin ^{-2} \theta Y_{, \varphi}^{l^{\prime} m^{\prime}} Y_{, \varphi}^{l m *}\right) d \Omega=l(l+1) \delta_{l l^{\prime}} \delta_{m m^{\prime}} \\
\int\left[\left(D_{2} Y^{l^{\prime} m^{\prime}}\right)\left(D_{2} Y^{l m *}\right)+\sin ^{-2} \theta\left(D_{1} Y^{l^{\prime} m^{\prime}}\right)\left(D_{1} Y^{l m *}\right)\right] d \Omega=\lambda l(l+1) \delta_{l l^{\prime}} \delta_{m m^{\prime}}
\end{gathered}
$$

where the integration is carried over a 2 -sphere $r=$ const, an asterisk denotes complex conjugation, and $\delta_{n n^{\prime}}$ is the Kronecker delta.

\section{APPENDIX B: SEPARATION OF THE FIELD EQUATIONS}

The field equations (15), which are fully separated with respect to $l, m$, and uncoupled at their principle part with respect to $i$, are obtained by substituting both expansions (8) and (12) into Eq. (4), and then considering certain combinations of the resulting equations and derivatives thereof. The necessary combinations are given in table III.

\begin{tabular}{c|c}
\hline \hline To get equation for ... & use the combination... \\
\hline \hline$i=1$ & $\{t t\}+f^{2}\{r r\}$ \\
$i=2$ & $\{t r\}$ \\
$i=3$ & $\{t t\}-f^{2}\{r r\}$ \\
$i=4$ & $(s\{t \theta\})_{,}+\left(s^{-1}\{t \varphi\}\right)_{, \varphi}$ \\
$i=5$ & $(s\{r \theta\})_{,}+\left(s^{-1}\{r \varphi\}\right)_{,}$ \\
$i=6$ & $\{\theta \theta\}+s^{-2}\{\varphi \varphi\}$ \\
$i=7$ & $s^{-2} D_{1}[s\{\theta \varphi\}]+s^{-1} D_{2}\left[s^{2}\{\theta \theta\}-\{\varphi \varphi\}\right] / 2$ \\
$i=8$ & $\{t \theta\}, \varphi-\{t \varphi\}, \theta$ \\
$i=9$ & $\{r \theta\}, \varphi-\{r \varphi\}, \theta$ \\
$i=10$ & $s^{-2} D_{1}\left[s^{2}\left(\{\theta \theta\}-s^{-2}\{\varphi \varphi\}\right)\right] / 2-s^{-1} D_{2}[s\{\theta \varphi\}]$ \\
\hline \hline
\end{tabular}

TABLE III. Component combinations used in separating the perturbation equations. Here $s \equiv \sin \theta$, and the angular differential operators $D_{1}$ and $D_{2}$ are those defined in Eqs. (A3). Curely brackets represent Schwarzschild components of Eq. (4); thus, " $\{t t\}+f^{2}\{r r\}$ ", for example, stands for "take the $t t$ component of Eq. (4), and add to it the $r r$ component of that equation, multiplied by $f^{2}$."

\section{APPENDIX C: MODE-DECOMPOSED LINEARIZED EINSTEIN EQUATIONS IN THE LORENZ GAUGE}

We give here the explicit expressions for the terms $\tilde{\mathcal{M}}_{(j)}^{(i)}$ appearing in the original form of the separated field equations, Eqs. (15). For the numerical evolution in this paper we use a different form of the separated equations [i.e., Eqs. (17), with Eqs. (18), which incorporate a constraint damping scheme], and so the original functions $\tilde{\mathcal{M}}_{(j)}^{(i)}$ are not needed in our analysis. We nevertheless give them here, as they might be useful as a starting point for anyone wishing to implement a different numerical scheme. The terms $\tilde{\mathcal{M}}_{(j)}^{(i)}$ are given by

$$
\begin{gathered}
\tilde{\mathcal{M}}_{(j)}^{(1)} \bar{h}^{(j)}=\frac{1}{2} f f^{\prime} \bar{h}_{, r}^{(1)}-\frac{1}{2} f^{\prime} \bar{h}_{, t}^{(2)}+\frac{f^{2}}{2 r^{2}}\left(\bar{h}^{(1)}-\bar{h}^{(3)}-\bar{h}^{(5)}-f \bar{h}^{(6)}\right), \\
\tilde{\mathcal{M}}_{(j)}^{(2)} \bar{h}^{(j)}=\frac{1}{2} f f^{\prime} \bar{h}_{, r}^{(2)}-\frac{1}{2} f^{\prime} \bar{h}_{, t}^{(1)}+\frac{f^{2}}{2 r^{2}}\left(\bar{h}^{(2)}-\bar{h}^{(4)}\right), \\
\tilde{\mathcal{M}}_{(j)}^{(3)} \bar{h}^{(j)}=\frac{1}{2} f f^{\prime} \bar{h}_{, r}^{(3)}+\frac{1}{2 r^{2}}\left[1-8 M / r+10(M / r)^{2}\right] \bar{h}^{(3)}-\frac{f^{2}}{2 r^{2}}\left[\bar{h}^{(1)}-\bar{h}^{(5)}-(1-4 M / r) \bar{h}^{(6)}\right], \\
\tilde{\mathcal{M}}_{(j)}^{(4)} \bar{h}^{(j)}=\frac{1}{4} f^{\prime} f \bar{h}_{, r}^{(4)}-\frac{1}{4} f^{\prime} \bar{h}_{, t}^{(5)}-\frac{3}{4} f^{\prime}(f / r) \bar{h}^{(4)}-\frac{1}{2} l(l+1)\left(f / r^{2}\right) \bar{h}^{(2)},
\end{gathered}
$$




$$
\begin{gathered}
\tilde{\mathcal{M}}_{(j)}^{(5)} \bar{h}^{(j)}=\frac{1}{4} f f^{\prime} \bar{h}_{, r}^{(5)}-\frac{1}{4} f^{\prime} \bar{h}_{, t}^{(4)}+\frac{f}{r^{2}}(1-3.5 M / r) \bar{h}^{(5)}-\frac{f}{2 r^{2}} l(l+1)\left(\bar{h}^{(1)}-\bar{h}^{(3)}-f \bar{h}^{(6)}\right)-\frac{f^{2}}{2 r^{2}} \bar{h}^{(7)}, \\
\tilde{\mathcal{M}}_{(j)}^{(6)} \bar{h}^{(j)}=-\frac{f}{2 r^{2}}\left[\bar{h}^{(1)}-\bar{h}^{(5)}-(1-4 M / r)\left(f^{-1} \bar{h}^{(3)}+\bar{h}^{(6)}\right)\right], \\
\tilde{\mathcal{M}}_{(j)}^{(7)} \bar{h}^{(j)}=-\frac{f}{2 r^{2}}\left(\bar{h}^{(7)}+\lambda \bar{h}^{(5)}\right), \\
\tilde{\mathcal{M}}_{(j)}^{(8)} \bar{h}^{(j)}=\frac{1}{4} f^{\prime} f \bar{h}_{, r}^{(8)}-\frac{1}{4} f^{\prime} \bar{h}_{, t}^{(9)}-\frac{3}{4} f^{\prime}(f / r) \bar{h}^{(8)} \\
\tilde{\mathcal{M}}_{(j)}^{(9)} \bar{h}^{(j)}=\frac{1}{4} f^{\prime}\left(f \bar{h}_{, r}^{(9)}-\bar{h}_{, t}^{(8)}\right)+\frac{f}{r^{2}}\left[(1-3.5 M / r) \bar{h}^{(9)}-(f / 2) \bar{h}^{(10)}\right] \\
\tilde{\mathcal{M}}_{(j)}^{(10)} \bar{h}^{(j)}=-\frac{f}{2 r^{2}}\left(\bar{h}^{(10)}+\lambda \bar{h}^{(9)}\right),
\end{gathered}
$$

where we have omitted all indices $l, m$ for brevity.

[1] E. Poisson, Living Rev. Relativity, 7, 6 (2004).

[2] Summary of the current status and recent progress on the problem can be found in a special issue of Classical and Quantum Gravity: Gravitational radiation from binary black holes: Advances in the perturbative approach, ed. C. O. Lousto [Class. Quantum Grav. 22, no. 15 (2005)].

[3] L. Barack and C. Cutler, Phys. Rev. D 69, 082005 (2004).

[4] T. C. Quinn and R. M. Wald, Phys. Rev. D 56, 3381 (1997).

[5] Y. Mino, M. Sasaki, and T. Tanaka, Phys. Rev. D 55, 3457 (1997).

[6] S. Detweiler and B. F. Whiting, Phys. Rev. D, 67024025 (2003).

[7] The mode sum scheme for SF calculations has been suggested in L. Barack and A. Ori. Phys. Rev. D 61, 061502R (2000). The full set of "regularization parameters" (necessary for implementing the scheme), for any orbits in Schwarzschild, was published in L. Barack, Y. Mino, H. Nakano, A. Ori, and M. Sasaki, Phys. Rev. Lett. 88, 091101 (2002). The regularization parameters for all orbits in Kerr were obtained in L. Barack and A. Ori, Phys. Rev. Lett. 90, 111101 (2003).

[8] L. Barack and A. Ori, Phys. Rev. D 64, 124003 (2001).

[9] T. Regge and J. A. Wheeler, Phys. Rev. 108, 1063 (1957).

[10] F. J. Zerilli, J. Math. Phys. 11, 2203 (1970).

[11] F. J. Zerilli, Phys. Rev. D 2, 2141 (1970).

[12] V. Moncrief, Ann. Phys. 88, 323 (1974).

[13] C. O. Lousto, Class. Quantum Grav. 22, number 15, S569 (2005).

[14] P. L. Chrzanowski, Phys. Rev. D 11, 2042 (1975).

[15] S. A. Teukolsky, Phys. Rev. Lett. 29, 1114 (1972).

[16] R. M. Wald, Phys. Rev. Lett. 41, 203 (1978).

[17] A. Ori, Phys. Rev. D 67, 124010 (2003).

[18] Y. Mino, Prog. Theor. Phys. 99, 79 (1998); Y. Mino and H. Nakano, ibid, 100507 (1998); H. Nakano and M. Sasaki, ibid 105, 197 (2001).

[19] S. Detweiler, Class. Quant. Grav. 22, S681 (2005).

[20] C. O. Lousto, Phys. Rev. Lett. 84, 5251 (2000).

[21] L. Barack and C. O. Lousto, Phys. Rev. D 66, 061502R (2002).

[22] L. Lindblom, M. A. Scheel, L. E. Kidder, H. P. Pfeiffer, D. Shoemaker, and S. A. Teukolsky, Phys. Rev. D, 69 124025 (2004).

[23] F. Pretorius, Class. Quant. Grav. 22, 425 (2005); F. Pretorius (submitted), gr-qc/0507014.

[24] C. W. Misner, K. S. Thorne, and J. A. Wheeler, Gravitation (Freeman, San Francisco, 1973).

[25] R. M. Wald, general relativity (The University of Chicago Press, Chicago and London, 1984). 
[26] For a comprehensive review of tensor multipole expansions, see K. S. Thorne, Rev. Modern Phys. 52, 299 (1980).

[27] L. Barack, Phys. Rev. D 64, 084021 (2001).

[28] C. Gundlach, G. Calabrese, I. Hinder, and J. M. Martin-García, Class. Quant. Grav. 22, 3767 (2005).

[29] S. Detweiler and E. Poisson, Phys. Rev. D 69, 084019 (2004).

[30] E. Poisson, Phys. Rev. D 52, 5719 (1995); Phys. Rev. D 55, 7980 (1997).

[31] K. Glampedakis and D. Kennefick, Phys. Rev. D 66, 044002 (2002).

[32] S. A. Hughes, Phys. Rev. D 61, 084004 (2000).

[33] C. O. Lousto and R. H. Price, Phys. Rev. D 56, 6439 (1997).

[34] L. Barack and L. M. Burko, Phys. Rev. D 62, 084040 (2000).

[35] K. Martel and E. Poisson, Phys. Rev. D 66, 084001 (2002).

[36] C. O. Lousto, Class. Quantum Grav. 22, number 15, S543 (2005).

[37] K. Martel, Phys. Rev. D 69, 044025 (2004).

[38] M. J. Pfenning and E. Poisson, Phys. Rev. D 65, 084001 (2002).

[39] R. A. Isaacson, Phys. Rev. 166, 1272 (1968).

[40] W. Krivan, P. Laguna, P. Papadopoulos, and N. Andersson, Phys. Rev. D 56, 3395 (1997).

[41] E. Pazos-Avalos and C. O. Lousto (submitted), gr-qc/0409065.

[42] G. N. Hernández-Warren, unpublished thesis, University of Texas at Brownsville (2005). 